Research Article

\title{
Investigation of an Underwater Vectored Thruster Based on 3RPS Parallel Manipulator
}

\author{
Tao Liu, ${ }^{1}$ Yuli Hu $\mathbb{D D}^{2}$ and Hui Xu $\mathbb{D}^{1}$ \\ ${ }^{1}$ School of Marine Science and Technology, Northwestern Polytechnical University, Xi'an, China \\ ${ }^{2}$ Key Laboratory for Unmanned Underwater Vehicle, Northwestern Polytechnical University, Xi'an 710072, China \\ Correspondence should be addressed to Yuli Hu; yulihu001@gmail.com and Hui Xu; merleliu@mail.nwpu.edu.cn
}

Received 24 July 2020; Revised 5 September 2020; Accepted 11 September 2020; Published 29 September 2020

Academic Editor: Mohamed El Ghami

Copyright (C) 2020 Tao Liu et al. This is an open access article distributed under the Creative Commons Attribution License, which permits unrestricted use, distribution, and reproduction in any medium, provided the original work is properly cited.

\begin{abstract}
Autonomous underwater vehicles (AUVs) are important and useful tool platforms in exploring and utilizing ocean resource. However, the effect of control surfaces would decrease even invalid complete in this condition, and it is very hard for conventional AUVs to perform detailed missions at a low forward speed. Therefore, solving this problem of AUVs becomes particularly important to increase the application scope of AUVs. In this paper, we present a design scheme for the vectored thruster AUV based on 3RPS parallel manipulator, which is a kind of parallel manipulator and has advantages of compact structure and reliable performance. To study the performance and characteristics of the proposed thrust-vectoring mechanism, a series of works about corresponding kinematic and dynamic analysis have been performed through the theoretical analysis and numerical simulation. In the part of kinematics, the inverse, forward kinematics, and workspace analysis of the thrust-vectoring mechanism is presented, and the numerical simulations are accomplished to prove the feasibility and effectiveness of this design in AUVs. In order to further verify feasibility of the thrust-vectoring mechanism, based on the considerations of various affecting factors, a dynamic model of the designed thrust-vectoring mechanism is established according to theoretical analysis, and the driving forces of the linear actuator are presented through a series of numerical simulations. In addition, a control scheme based on PID algorithm is proposed for the designed vectored thruster with considering various affecting factors and the application environment. Meanwhile, the control scheme is also established and verified in MATLAB Simscape Mutibody. A series of numerical simulations of the thrust-vectoring mechanism prove the feasibility of the vectored thruster. According to equipping the designed vectored thruster, the AUVs can overcome the limit of weakening the control ability at zero or low forward speeds, and this improvement also expands the application of it, which has been scaled greatly.
\end{abstract}

\section{Introduction}

Over the last few decades, due to exhausting of resources and energy, human beings are bearing with a series of survival predicaments and development challenges $[1,2]$. Because of the lack of land-based resource and the continuing need for all kinds of resources, an increasing number of countries and scientists have paid more and more attention on the exploitation and utilization of resources [3-5]. In the present, most of the water available on Earth exists in the oceans, yet only a small part of this vast resource has been explored [2]. The ocean has vast areas and is rich in all kinds of natural resources, such as marine life, oil, natural gas, and minerals. Additionally, the ocean not only contains a lot of marine recourse but also brings a lot of traffic convenience around the world. With the progress of society and economy, the development of mineral resource has become an inevitable trend. Exploring and exploiting the oceans has become the principal development strategy of every country in the world. However, the nature environment of the ocean is too harsh to explore, and the advanced technology has been rapidly developed in recent years, such as autonomous underwater vehicles (AUVs), remotely operated vehicles (ROVs) [6-8], and unmanned marine vehicles (USVs) [9]. In addition, other different techniques have also been used for controlling all kinds of robots, such as proportional integral derivate [10], fuzzy control [11, 12], and sliding model control [13]. 
AUVs have become a main tool for surveying below the sea due to the great improvement in their performance and advancement in underwater research. Through equipping a large quantity of advanced instruments and equipment, AUVs are capable of accomplishing applications including scientific, commercial, and military tasks such as exploration of oceans $[14,15]$, oceanography mapping surveys [16-18], the collecting ocean environment information [19-21], and searching and rescuing for shipwrecks $[22,23]$ and debris from the missing airplanes $[24,25]$. With the expanding area of applications, the design of AUVs needs to meet the higher demand continuously. Although it has made great steps in AUVs performance, the new emerging technologies and demands for exploiting oceans have attracted critical mass of scientists and engineers to undertake the research of AUVs.

Conventional AUVs are designed equipped with a main propeller and control surfaces at the tail cone for propulsion and control [26-28]. These conventional AUVs can fulfill the work well under normal conditions. When the conventional AUVs need to complete exploration tasks with a lower speed in a complex and unknown underwater environment, the control capability of AUV depends heavily on the control faces made up of fin and rudder. The velocity of AUV is relatively low or zero because of the demand of practical problems, such as scanning topographic map, taking photographs, and monitoring marine observation data. However, they are unable to perform detailed inspection missions at zero or low forward speeds due to the control faces which become ineffective in this condition $[28,29]$. The cause of this problem is that the generation of control forces from control surfaces depends on forward speeds of AUVs $[28,30]$. Therefore, this disadvantage has further development and application of conventional AUVs greatly.

There are some approaches to solve this problem, such as installing additional thrusters to provide additional control forces for controlling AUVs [28, 31-34], but this method would result in the problems of complicated structure and increasing energy consumption. Its complex structure, adverse working environment, and so on causes the decrease of reliability of the whole AUV control system. Installation and maintenance of additional thrusters would significantly increase energy expenditure or energy carrier for sailing.

Another more efficient and workable method to release this restriction is to use vectored thruster to replace the conventional propulsion types [7, 29, 35-38]. The AUVs equipped with vectored thruster do not require the use of fin and rudder for controlling at all. Since this kind of AUVs driven by a vectored thruster, the control forces are generalized force components produced by vectored thruster, and these forces only depend on the rotational speed of the propeller. Therefore, the AUVs equipped with vectored thruster are independent from any control forces generated by control faces, and the controllability of vehicle is markedly improved and obtains a better good application effect. So, the vectored thruster AUVs are capable of accomplishing detailed missions at a low forward speed.

In the research areas of this field, some companies and research institutions have made progress in theory study and application of vectored thrusters $[29,36,38]$. Among above research AUVs, Bluefin and MBARI have achieved great successes and provided considerable experience in the use and study of vectored thrusters. More importantly, the engineering practice of Bluefin and MBARI shows that this method can raise control efficiency greatly and also reduce the possibility of losing control at low speed. However, the existing design of the vectored thruster is almost designed based on serial mechanism; this kind of mechanism has the disadvantages of complex structure, low bearing capacity, and high moment inertia. So, based heavily on practicalities of serial mechanism, the existing design of the vectored thruster is too bulky and complex to use for AUVs. Considering the restrictions of application environment and structure size, it is crucial to choose a suitable mechanism for designing new vectored thruster AUVs.

On the contrary, compared with other commonly used mechanical structures, parallel manipulators have numerous advantages, such as small size, compact and reasonable structure, reliable performance, fast response, high positioning precision, high stability, high sensitivity, high stiffness, and better dynamic performance [39-42]. Those merits of parallel manipulators make the device have high popularization value and use value, such as medical and industrial robots, flight simulator, and mechanical device. Inspired by various applications of parallel manipulators, the idea of vectored thruster AUVs based on parallel manipulator is generated. In the field of thrust-vectoring mechanism research, many scientists and engineers have made great contributions to the development of vectored thruster AUVs based on parallel manipulators. The full deflection vectored thruster is based on the spatial linkage and universal joint proposed by Cavallo and Michelini [43]; the authors designed a 3-SPS-S parallel manipulator with passive constraining spherical joints to drive the underwater vehicle [30]. The above thrusters currently have some problems, such as the structure is relatively complex, and the motion real-time resolving method and the dynamics model for the vectored thruster are difficult.

With comparing structure characteristics of different kinds of parallel manipulators and considering actors of application environment, 3RPS is chosen from various parallel manipulators as the thrust-vectoring mechanism mainly various advantages, including its compact structure, high position tracking precision, and fast response speed. This parallel mechanism is a strong coupled nonlinear structure, so its motion control is too complex to use more widely [44-47]. Despite its advantages, the 3RPS parallel manipulator also needs to overcome some problems that would restrict the development and application of the thrust-vectoring mechanism. Through reading and analyzing the domestic and foreign related literature, various methods of kinematics and dynamics for 3RPS parallel manipulators have been presented [44, 45, 48-50].

On the basis of the above considerations, the design concept of vectored thruster which is made up of 3RPS parallel manipulators is introduced. The vectored thruster based on 3RPS parallel manipulators has terse structure, convenient operation, convenient installation, steady working system, and wide adjustable range. Using this 
method, the AUVs are able to provide the vectored thrust effectively and efficiently. More than anything, the AUVs equipped with vectored thruster are able to complete a variety of the complex tasks at a comparably low forward speed.

In this paper, the structural design of the vectored thruster based on 3RPS is introduced briefly. In order to satisfy the design requirements and study the motion characteristics of vectored thruster, the kinematics and dynamics model of the thrust-vectoring mechanism are established, and the related simulation is presented to verify feasibility of the scheme. Finally, a control scheme for the vectored thruster is designed and simulated in Matlab. The theoretical analysis and numerical simulations prove that the proposed vectored thruster based on a 3RPS parallel manipulator can effectively realize the function of providing the required vectored thrust for thrust-vectoring propulsion.

\section{The General Design of Vectored Thruster}

The configuration of the whole AUV equipped with the designed vectored thruster based on 3RPS parallel manipulator is presented, as shown in Figure 1. Due to the existence of the vectored thruster, the AUVs do not need any more rudders to provide control forces. The force generated by the vectored thruster is used as control force for AUV's yaw and pitch motion. Consequently, the tilt angle is important one of the criteria to assessing the performance of the proposed vectored thruster. However, the space in the stern of AUVs is limited, and the tilt angle range of the designed vectored thruster is also limited. Referring to a literature review $[29,51]$, the duct propeller's tilt angle is limited to plus or minus $15^{\circ}$ in our design. In addition, the vectored thruster contains a duct that can be used for protecting the propeller form damaging and enhancing flow capability.

In terms of structure design, considering the specific requirements of application environment and the stability of system, we adopted the modular design for vectored thruster AUV. The designed vectored thruster is mounted on the stern of an AUV as an integral and independent, which is adopted for convenient installation and maintenance. The designed vectored thrust duct propeller system mainly contains the duct propeller and the thrust-vectoring mechanism. A whole structure model of the vectored thruster AUV based on 3RPS parallel manipulator is built up, as shown in Figure 2.

At present, the duct propeller is the most widely used form of propulsion device for underwater robots. A duct propeller is mainly composed of an annular wing and a propeller. There are many underwater vehicles equipped with duct propellers, for the extraordinary performance of improving the propulsive efficiency and avoiding cavitation conditions [52]. This kind of propeller is able to provide the thruster from zero to cruising speed more effectively. Just because of an effectively increased thrust in the condition of a low forward speed, the duct propeller is widely used in various marine vessels, such as AUVs and ROVs.

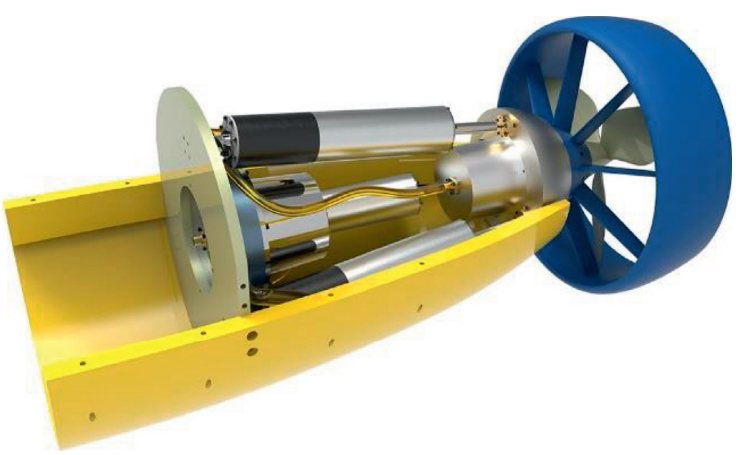

FIgURE 1: Vectored thruster mounted at AUV.

Furthermore, underwater environments are very complex and harsh; propellers are very easily destroyed by underwater animals and plants, waves, even currents, and other uncertainties. Hence, the existence of a duct can protect the propeller against damage from the underwater environment during all kinds of missions. Moreover, since the duct also can generate the thrust during the voyage, the duct is an important source of control force for AUV's yaw and pitch motion.

In our design, the duct propeller is driven by a main electric motor installed in the rotating body, which is aligned with the holes of the duct's inner shaft with fastening screws. In order to simplify the unnecessary transmission structure and reduce the redundant weight, the main motor has been bedded on the rotating body with a duct propeller. It is very clean and efficient to take direct connects with the propeller and the main motor. This installation mode of the main motor and rotating body can improve space utilization significantly and reduce the weight of vectored thruster effectively. And this modularity makes the duct propeller system easy to maintain and debug on the whole vectored thruster control system.

As the implementing actuator of the vectored thruster, the thrust-vectoring mechanism is fundamental to the overall system for its basic functions. There are many methods on how to realize thrust vectoring, and each method has its own advantages and disadvantages. Considering the limited space of AUVs' tailcone and the harsh operation condition, it is central to choose an appropriate mechanism structure that can complete the design function of achieving vector control effectively for AUVs. Comparing to the serial mechanism, parallel manipulators have many inherent superiorities, such as small size, compact and reasonable structure, reliable performance, fast response, high positioning precision, high stability, high sensitivity, high stiffness, and better dynamic performance.

Integrating practical application environment of AUVs and based on the application background of various parallel manipulators, 3RPS parallel manipulator is chosen as the thrust-vectoring mechanism after analyzing various mechanical structures. In accordance with this notion, a novel thrust-vectoring mechanism based on the 3RPS parallel manipulator for AUVs is designed, as shown in Figure 3.

The thrust-vectoring mechanism is designed based on 3RPS manipulator, which has a top rotting platform, a fixed base, and three identical sets of driving limbs and joints. 


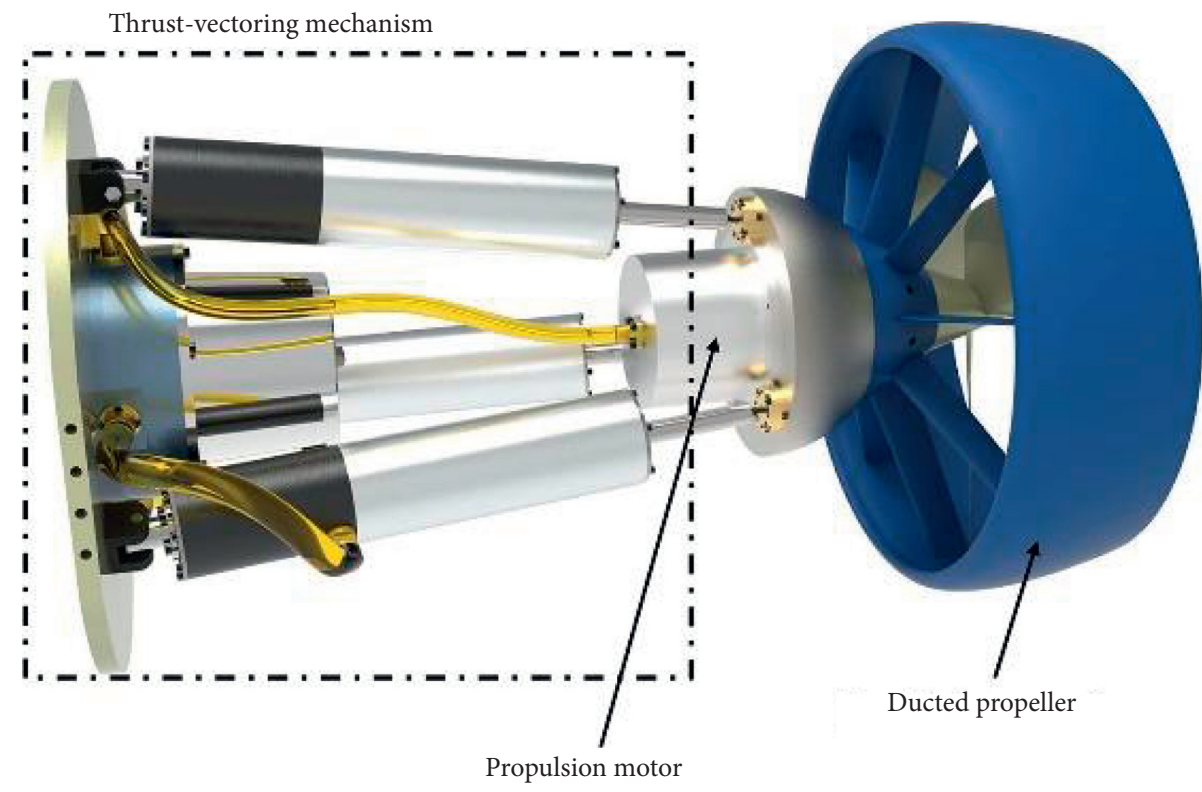

FIGURE 2: Vectored duct thrust propeller.

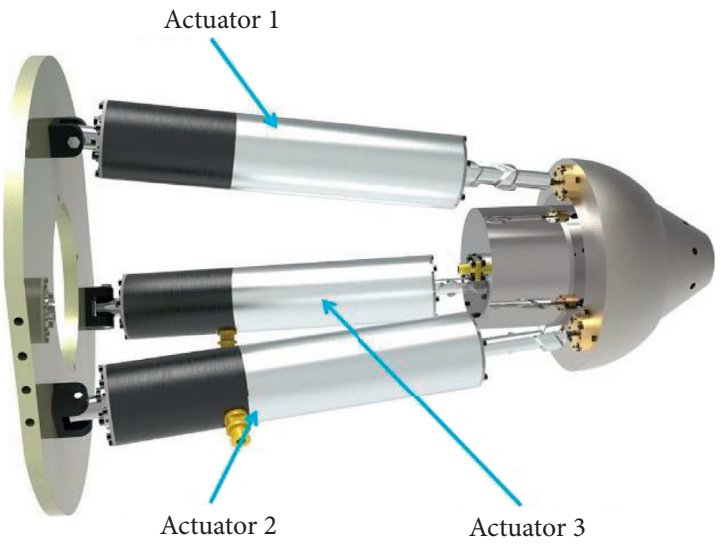

FIgURE 3: Thrust-vectoring mechanism based on 3-RPS parallel manipulator.

Each driving limb has an actuating prismatic joint $(P)$ attached to the fixed base by a rotational joint $(R)$ and connected to the platform by a spherical joint $(S)$ [53-55]. Each limb is driven by a linear actuator. Thus, the length of limb could be changed within the operation range, and the top platform would rotate when the length of limbs changes with a certain law of motion. The vectored thruster is installed on the tail horizontally, which connected with the shell of the AUV via fastening screws. The duct propeller can rotate around the center of the top platform horizontally and vertically, and the thrust generated by the vectored thruster will drive the vehicle forward or changes the direction of movement.

The 3RPS parallel manipulator has two rotational and one translational degree of freedom (DOF). It is superfluous to have the translational DOF for the thrust-vectoring mechanism, the redundant DOF needs to be constrained by motion control, and the other two rotational DOFs are used to realize functions of the thrust-vectoring mechanism. In addition, the translational DOF of 3RPS parallel manipulator will make the top rotating body bump into the shell of the AUV. So, the importance of the study on redundant DOF of the 3RPS parallel manipulator is obvious for the actual application of the thrustvectoring mechanism.

Since the vectored thruster could generate required control forces for controlling AUVs motion, there is no need to have extra rudders as conventional AUVs. The component of the thrust as control forces is dependent on the deflection angle and the thrust of the vectored thruster. Therefore, the research on deflection angle of the vectored thruster is essential for controlling the motions of AUVs. However, it is very difficult to measure the tilt angle of the vectored thruster directly because the limited space and underwater environment is not suitable for installing sensors to measure. Another common and efficient approach to get the rotation angles is using the kinematic analysis method, which can obtain the tilt angle by measuring the lengths of the three limbs. Based on this kinematic method, tilt angle information about the vectored thruster can be obtained via relative calculation with the lengths of three limbs, which can be measured directly by length sensors installed in actuators.

In order to realize precision and stable positioning control of the proposed vectored thruster, the design of the automation control system is fundamental to achieve objectives. Hence, establishment of kinematic and dynamic models for the thrust-vectoring mechanism based on the 3RPS parallel manipulator is significant to achieving perfect control of the vectored thruster based on the above analysis.

\section{Kinematic Analysis of the Thrust- Vectoring Mechanism}

The thrust-vectoring mechanism is designed based on the 3RPS parallel manipulator, which is composed of a base plate, a rotating platform, and three uniformly distributed driving 
limbs, as shown in Figure 3. According to the needs of analyzing the motion of the top rotating platform, two Cartesian coordinate systems with associated symbols have been established in the 3RPS parallel manipulator and shown in Figure 4. The reference frame $O-x y z$, which is the global coordinate system, is fixed to the center of the immovable base and the $z$-axis normal to the fixed base. Similarly, the reference frame P-ijk denotes the local coordinate system located on center point $P$ of the rotating platform, whose $j$-axis is perpendicular to the bottom surface of the platform.

The moving sides of driving limbs (linear actuators) are connected to the upper rotating platform through three sphere joints that is fixed directly to the center of the top platform, while the other sides of the limbs are connected to the base with three revolute joints that are symmetrical about the center of base. $A_{1}, A_{2}$, and $A_{3}$ are the connected points between the fixed base and the driving legs (linear actuators), $B_{1}, B_{2}$, and $B_{3}$ denote the points of the revolute joints. It should be mentioned that $A_{1} B_{1}, A_{2} B_{2}$, and $A_{3} B_{3}$ are perpendicular to the fixed base because $A_{1} B_{1}, A_{2} B_{2}$, and $A_{3} B_{3}$ represent revolute joints with a certain height. A reference frame $O^{\prime}-x y z$ is established with respect to the plane formed by points $B_{1}, B_{2}$, and $B_{3}$, and this plane parallels with the fixed base $A_{1}, A_{2}$, and $A_{3}$. The connected points between the moving parts and the rotating platform are expressed as $C_{1}$, $C_{2}$, and $C_{3}$. The radius of the fixed base and the top platform are defined as $r_{1}$ and $r_{2}, O$ and $P$ denote the center points of the base and top platform, respectively. $L_{1}, L_{2}$, and $L_{3}$ denote the lengths of three linear actuators between the top platform and the fixed base.

As we can see in Figure 4, in the global reference frame $O-x y z$, the center point of the equilateral triangle made up of three points $A_{1}, A_{2}$, and $A_{3}$ is expressed as $O$, and the radius of the fixed base is defined as $O A_{1}=O A_{2}=O A_{3}=r_{1}$. Hence, the location of $A_{i}$ in global reference frame $O-x y z$ can be described as follows:

$$
P_{A-O}=\left[\begin{array}{lll}
A_{1} & A_{2} & A_{3}
\end{array}\right]=\left[\begin{array}{ccc}
0 & r_{1} & -r_{1} \\
-\frac{\sqrt{3} r_{1}}{2} & \frac{r_{1}}{2} & \frac{r_{1}}{2} \\
0 & 0 & 0
\end{array}\right] .
$$

Similarly, $B_{1}, B_{2}$, and $B_{3}$ denote the axes of revolution of the revolute joints with a certain height $h_{r}$, and this plane parallel is with the fixed base. Hence, the locations of point $B_{i}$ can be denoted as follows:

$$
P_{B-O}=\left[\begin{array}{lll}
B_{1} & B_{2} & B_{3}
\end{array}\right]=\left[\begin{array}{ccc}
0 & r_{1} & -r_{1} \\
-\frac{\sqrt{3} r_{1}}{2} & \frac{r_{1}}{2} & \frac{r_{1}}{2} \\
h_{r} & h_{r} & h_{r}
\end{array}\right] .
$$

A local coordinate system $P$-ijk is established on the rotating platform bottom surface of the thrust-vectoring mechanism, in which the origin point $P$ is the circumcenter of triangle $C_{1}, C_{2}$, and $C_{3}$. So, the locations of connection point between the linear actuators and the top platform can be described as follows:

$$
P_{C-P}=\left[\begin{array}{lll}
B_{1} & B_{2} & B_{3}
\end{array}\right]=\left[\begin{array}{ccc}
0 & r_{2} & -r_{2} \\
-\frac{\sqrt{3} r_{2}}{2} & \frac{r_{2}}{2} & \frac{r_{2}}{2} \\
0 & 0 & 0
\end{array}\right] .
$$

From Figure 4, $p$ as a position vector denotes the translation vector from the center point $O$ to point $P$ of top rotating platform in global reference frame $O-x y z$. To describe the relative motion between top rotating platform and fixed base, a rotation matrix about frame $P$-ijk with respect to the fixed base reference frame $O-x y z$ needs to be established. The position vector $p$ and the rotation matrix $R$ are defined as follows:

$$
\begin{aligned}
p & =\left[\begin{array}{lll}
P_{x} & P_{y} & P_{z}
\end{array}\right]^{T}, \\
R & =\left[\begin{array}{ccc}
c \gamma c \beta & c \gamma s \beta s \alpha-s \gamma c \alpha & c \gamma s \beta c \alpha+s \gamma s \alpha \\
s \gamma c \beta & s \gamma s \beta s \alpha+c \gamma c \alpha & s \gamma s \beta c \alpha-c \gamma s \alpha \\
-s \beta & c \beta s \alpha & c \beta c \alpha
\end{array}\right],
\end{aligned}
$$

where $s(\cdot)=\sin (\cdot), c(\cdot)=\cos (\cdot)$, and $\alpha, \beta$, and $\gamma$ denote the rotation angles about the $k$-axis, $j$-axis, and $i$-axis, respectively.

The thrust-vectoring mechanism only needs two rotational DOFs to realize its functionality; the 3RPS parallel manipulator has one more translational DOF that is redundant. In order to present the condition of the top rotating platform of the thrust-vectoring mechanism, the rotation angles are also important parameters that need to be defined. According to the need of the thrust-vectoring mechanism, the displacement between centers of the base and the top platform are set as $h$. A generalized vector $q$ is established to describe the position and orientation of the top rotating platform in the global reference frame as follows:

$$
q=\left[\begin{array}{llllll}
P_{x} & P_{y} & P_{z} & \alpha & \beta & \gamma
\end{array}\right]^{T},
$$

where $P_{x}, P_{y}$, and $\gamma$ are associated with the rotation angles $\alpha$ and $\beta$. Based on $P_{x}=r_{2}(s \alpha s \beta c \gamma-c \alpha s \gamma), \quad P_{x}=r_{2}$ $(s \alpha s \beta c \gamma-c \alpha s \gamma), P_{x}=r_{2}(s \alpha s \beta c \gamma-c \alpha s \gamma), P_{x}=r_{2}(s \alpha s \beta c \gamma-$ $c \alpha s \gamma), P_{y}=\left(r_{2} / 2\right)[(c \beta c \gamma-s \alpha s \beta s \gamma)-c \alpha c \gamma], L_{P}=\sqrt{P_{x}^{2}+P_{y}^{2}}$, and $\gamma=\tan ^{-1}[s \beta s \alpha /(s \beta+c \alpha)]$, so $P_{x}$ and $P_{y}$ also can be written as follows:

$$
\left\{\begin{array}{l}
P_{x}=r_{2} \cdot \delta_{P x}, \\
P_{y}=r_{2} \cdot \delta_{P y}, \\
L_{P}=r_{2} \cdot \delta_{L P},
\end{array}\right.
$$

where $\delta_{P x}, \delta_{P y}$, and $\delta_{L y}$ denote the influence factor of $P_{x}, P_{y}$, and $L_{P}$. According to the application needs to be designed in 


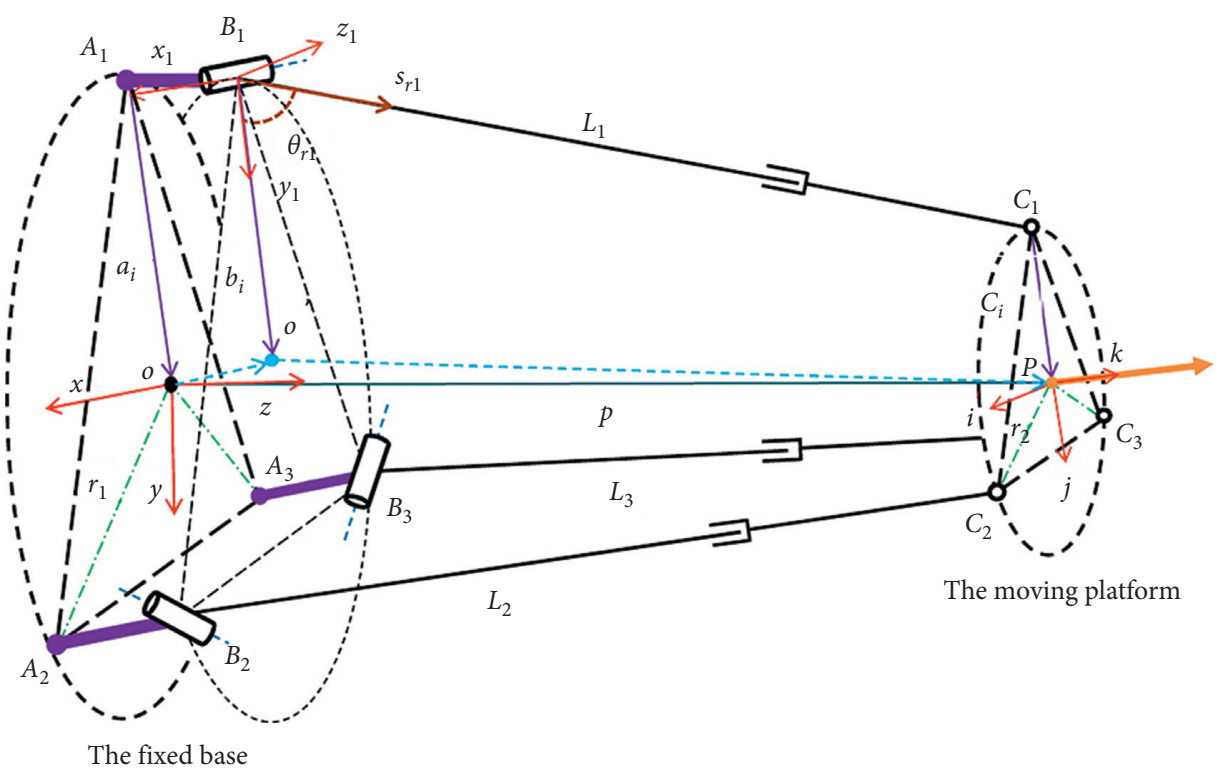

FIgURE 4: Schematic diagram of the 3-RPS parallel manipulator.

this design, the numerical simulations about $\delta_{P x}, \delta_{P y}$, and $\delta_{L P}$ and the angle of rotation of $\gamma$ can be calculated, and the simulation results are obtained and plotted in Figure 5.

From Sections 3.1 and 3.3, the inverse, forward kinematic analyses, and workspace analysis of the thrust-vectoring mechanism are performed, and numerical simulations are conducted to validate the accuracy and reliability accordingly.

3.1. Inverse Position Analysis of Thrust-Vectoring Mechanism. In this design, inverse position analysis of the thrust-vectoring mechanism is carried out to establish the mapping relations between the position and orientation of top moving platform and the lengths of three driving linear actuators. Referring to Figure 4, the length of linear actuator $L_{i}$ with respect to the fixed base reference frame $O-x y z$ can be written as

$$
L_{i}=\left|R c_{i}+p-a_{i}\right|
$$

where $a_{i}\left(c_{i}\right)$ denotes the vector from point $O(P)$ to point $A_{i}$ $\left(C_{i}\right)$ in frame $O-x y z(P-i j k)$.

The length change of the $i$ th limb can be calculated as

$$
\Delta L_{i}=L_{i}-L_{\text {ave }},
$$

where $L_{\text {ave }}$ is the initial length of the linear actuators at the tilt angle $\alpha=\beta=0^{\circ}$.

According to (7) and (8), the length changes of the three linear actuators can be obtained with related parameters presented in Table 1, and the dimension parameters of vectored thruster are calculated through CAD software. The results of the length change of $i$ th linear actuator are plotted in Figure 6.

To further study the relationship between the tilt angles of the top rotating platform and the lengths of three linear actuators, the top moving platform moves according to $\alpha_{s}=\pi / 9 \cdot \sin (t) \mathrm{rad}$ and $\beta_{s}=\pi / 9 \cdot \cos (t) \mathrm{rad}$. When the top rotating platform moves according to $\alpha=\alpha_{s}$ and $\beta=\beta_{s}$, based on the kinematic analysis of the thrustvectoring mechanism above, the length of linear actuators is plotted in Figure 7 .

3.2. Forward Position Analysis of Thrust-Vectoring Mechanism. Similarly, the forward position analysis of the thrust-vectoring mechanism is established to study the mapping relations between the lengths, three linear actuators, and the position and orientation of top moving platform. The position and orientation of the top moving platform is obtained according to the given length of the $i$ th linear actuator.

Referring to Figure 4, the position vector of point $C_{i}$ on the top rotating platform in global frame $O-x y z$ can be expressed as

$$
O C_{i}=O A_{i}+A_{i} B_{i}+B_{i} C_{i}
$$

where $B_{i} C_{i}$ denotes the vector of the $i$ th linear actuator, which can be expressed as

$$
\begin{gathered}
B_{i} C_{i}=\left[\begin{array}{c}
L_{i} c\left(\theta_{1 i}\right) c\left(\lambda_{i}\right) \\
L_{i} c\left(\theta_{1 i}\right) s\left(\lambda_{i}\right) \\
L_{i} s\left(\theta_{1 i}\right)+h_{r}
\end{array}\right], \\
\lambda_{i}=\frac{i \pi}{3}+\frac{\pi}{6},
\end{gathered}
$$

where $\theta_{1 i}$ is the angle between the actuator and the fixed base. Since points $C_{1}, C_{2}$, and $C_{3}$ form an equilateral triangle in the top rotating platform, based on the theory in geometry, the relationship of $C_{1} C_{2} C_{3}$ can be determined by

$$
\left|C_{1} C_{2}\right|=\left|C_{1} C_{3}\right|=\left|C_{2} C_{3}\right|=\sqrt{3} r_{2} .
$$




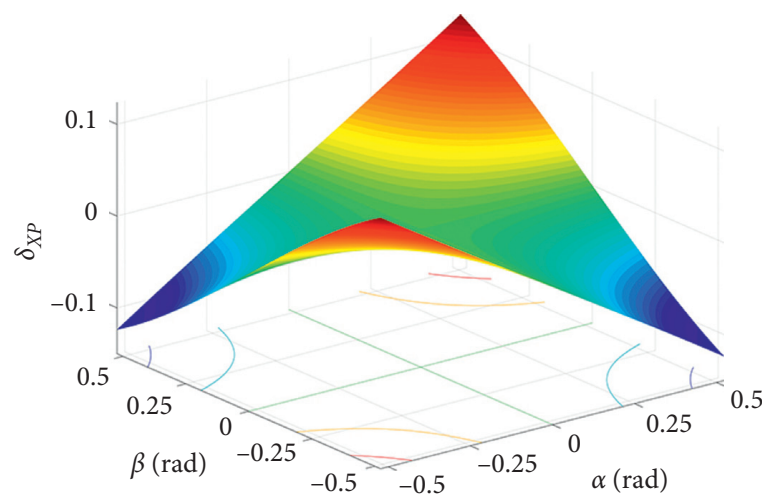

(a)

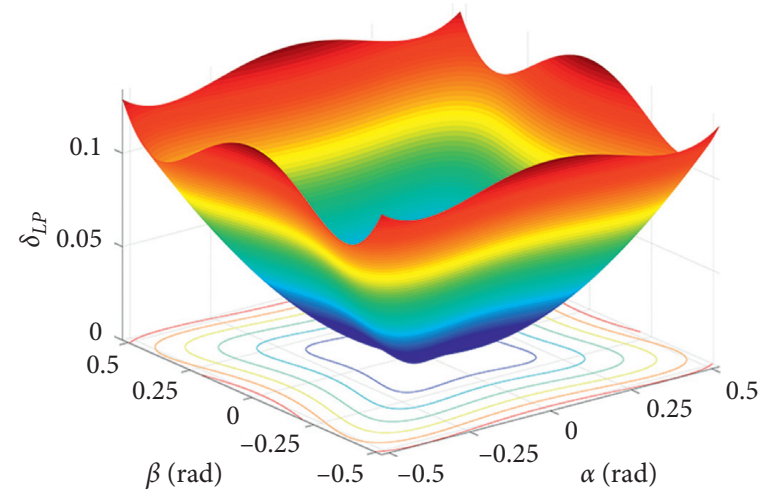

(c)

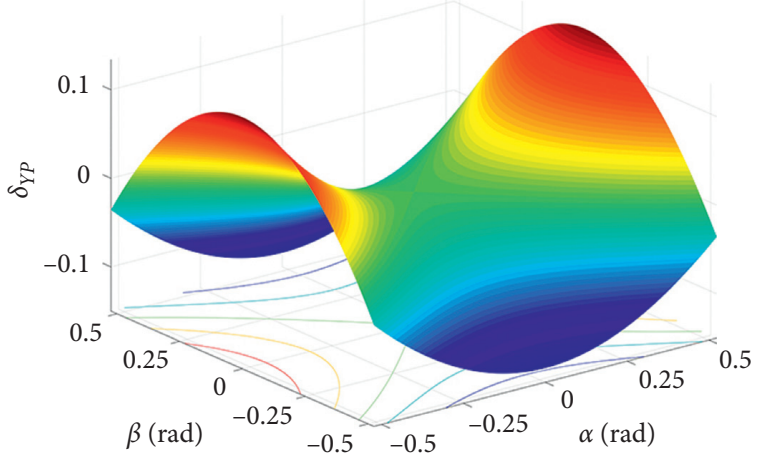

(b)

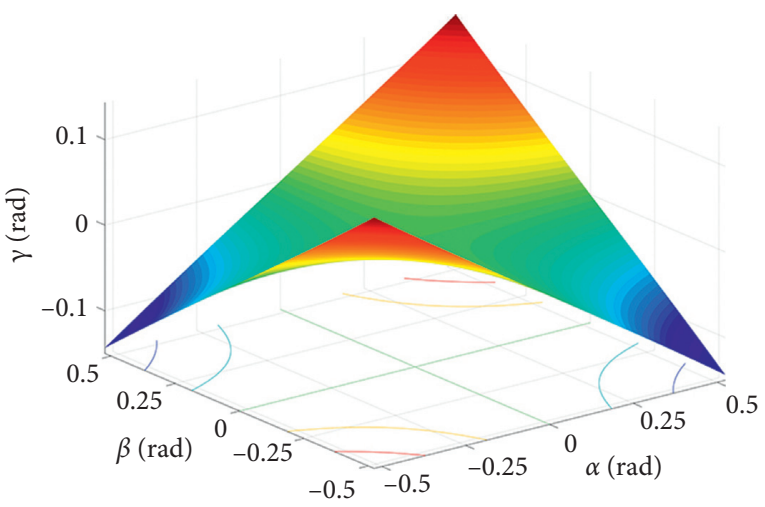

(d)

Figure 5: Influence factor.

TABle 1: Geometric Parameters of the thrust-vectoring mechanism.

\begin{tabular}{lc}
\hline Symbol & Value (unit) \\
\hline$r_{1}$ & $100(\mathrm{~mm})$ \\
$r_{2}$ & $60(\mathrm{~mm})$ \\
$h$ & $330(\mathrm{~mm})$ \\
$h_{r}$ & $10(\mathrm{~mm})$ \\
\hline
\end{tabular}

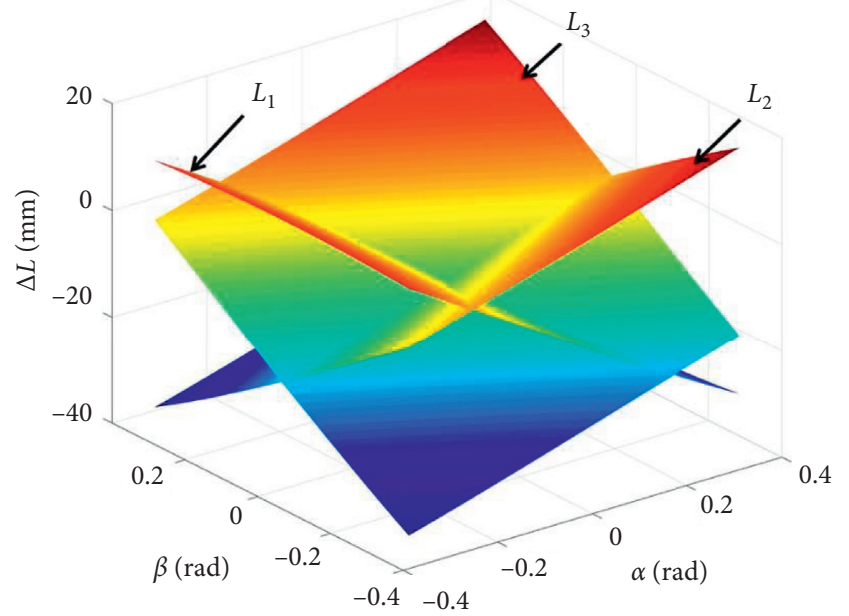

Figure 6: Length changes of linear actuators.
Hence, the position of points $C_{i}$ in reference frame $O-x y z$ can be obtained through (9)-(11).

Because $P$ is the center of the circumcircle generated by three points $C_{1} C_{2} C_{3}$ in the top moving platform, the position of center point $P$ in global reference frame $O-x y z$ can be expressed as

$$
\left|p-O C_{i}\right|=r_{2}
$$

where $p$ of the center point $P$ of the top rotating platform, which can be used to describe the position of point $P$ with three components $\left(P_{x}, P_{y}, P_{z}\right)$.

The position vector $O C_{i}$ of points $C_{i}$ is regarded as a known parameter when the length of linear actuator is given; thus, the three equations about the point of $P$ can be established and calculated.

To further investigate the relationship between the lengths of linear actuators with the position and orientation of the top platform, different lengths of the linear actuator are used for the forward kinematic analysis. In this simulation, the linear actuator $L_{1}, L_{2} \in\left[\begin{array}{ll}310 & 340\end{array}\right] \mathrm{mm}$ and $L_{3}=310$, $325,340 \mathrm{~mm}$. According to equations (9)-(12), the orientation and position vector of the top moving platform can be calculated directly, as shown in Figure 8.

3.3. Workspace Analysis of Thrust-Vectoring Mechanism. Due to the available space of AUV is limited, it is important to analyze the workspace of the thrust-vectoring 


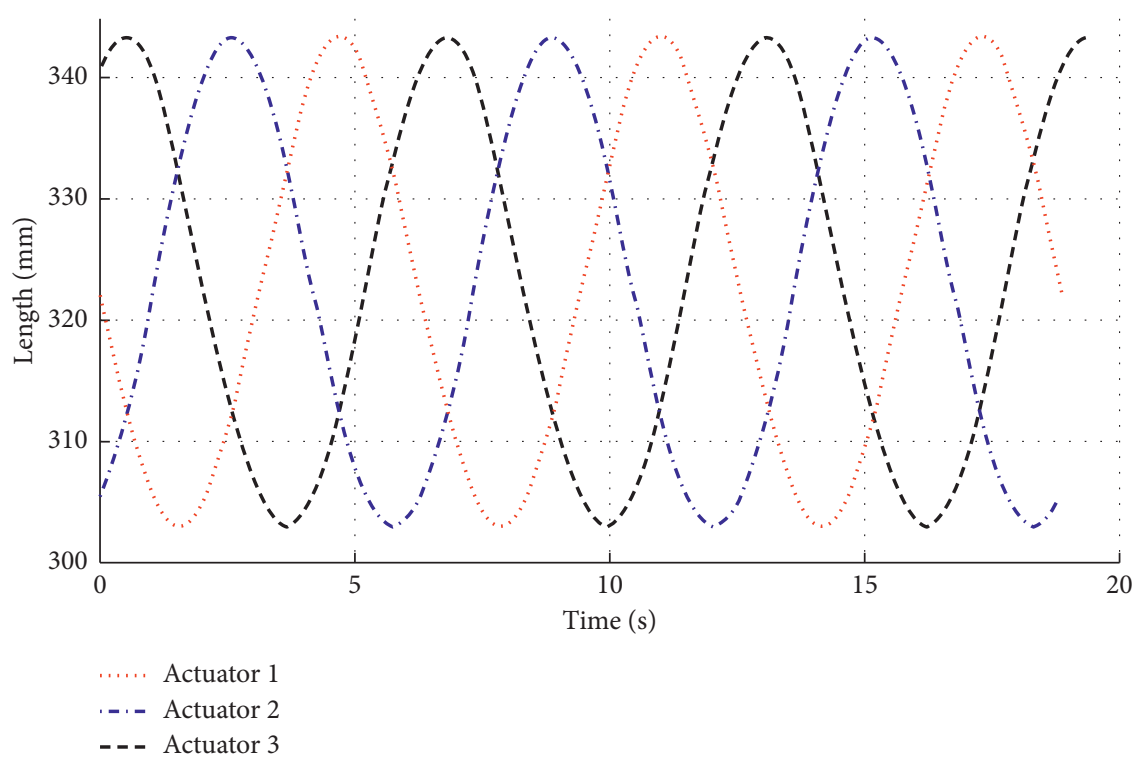

FIGURE 7: Length of linear actuators.

mechanism for optimizing structure design and improving performance. According to the kinematic analysis mentioned above, all the positions and orientations of the top rotating platform can be obtained by changing the lengths of actuators. Considering the motion characteristics of the thrust-vectoring mechanism and constraint on available space, the workspace analysis is mainly referring to study the tilt angle and angle change of the revolute joint and spherical joint of the thrust-vectoring mechanism in this paper.

In this section, $\theta_{r}$ and $\theta_{t}$ denote, respectively, the rotational angles of the revolute joint and spherical joint. The schematic diagram of revolute joint and spherical joint is presented, as shown in Figure 9.

The tilt angle $\theta_{r i}$ and angle change $\Delta \theta_{r i}$ of the revolute joint at point $B_{i}$ can be defined by

$$
\begin{aligned}
\theta_{r i} & =\arccos \frac{s_{r i} \cdot e_{1}}{\left|s_{r i}\right|\left|e_{1}\right|}, \\
\Delta \theta_{r i} & =\theta_{r i}-\theta_{r-\text { avg }},
\end{aligned}
$$

where $e_{1}$ is the direction vector of the $y$-axis in global reference frame $O-x y z$ and $\theta_{r-\text { avg }}$ is the initial angle generated by the linear actuator and the fixed base at the tilt angle $\alpha=\beta=0 \mathrm{rad}$.

The tilt angle $\theta_{t i}$ and angle change $\Delta \theta_{t i}$ of the spherical joint at point $C_{i}$ can be expressed as

$$
\begin{aligned}
\theta_{t i} & =\arccos \frac{s_{t i} \cdot t_{1}}{\left|s_{t i}\right|\left|t_{1}\right|}, \\
\Delta \theta_{t i} & =\theta_{t i}-\theta_{t-\text { avg }},
\end{aligned}
$$

where $t_{1}$ is the direction vector of the $k$-axis in local reference frame $P$-ijk and $\theta_{t \text {-avg }}$ is the initial angle between the linear actuators with the rotating platform at the tilt angle $\alpha=\beta=0 \mathrm{rad}$.
Similarly, to study the relations between the tilt angles of revolute and spherical joints and the lengths of linear actuators, the tilt angles of revolute joint $\theta_{r i}$ and spherical joint $\theta_{t i}$ by forward kinematic analysis are performed with linear actuator length $L_{1}, L_{2} \in\left[\begin{array}{ll}310 & 340\end{array}\right] \mathrm{mm}$ and $L_{3}=310,325$, $340 \mathrm{~mm}$. The simulation results are plotted in Figure 10.

The tilt angle of the three linear actuators and the top rotating platform has close relation, the platform moves according to $\alpha_{s}$ and $\beta_{s}$ mentioned above. When the top rotating platform moves according to $\alpha=\alpha_{s}$ and $\beta=\beta_{s}$ $\left(\alpha_{s}=\pi / 9 \cdot \sin \left(\pi \cdot(\mathrm{t} / 36) \mathrm{rad}\right.\right.$ and $\left.\beta_{s}=\pi / 9 \cdot \cos (\pi \cdot t / 36) \mathrm{rad}\right)$, the tilt angle and angle change of linear actuators are plotted in Figure 11.

\section{Dynamic Analysis of Thrust- Vectoring Mechanism}

For improving the dynamic performance and control accuracy of the designed vectored thruster, it is greatly important to analyze the dynamics model. Since the thrust-vectoring mechanism is designed based on the 3RPS parallel manipulator, which includes three closedloops kinematic chains, it is very complicated to perform the dynamic analysis of the thrust-vectoring mechanism.

According to the theoretical analysis and practical needs, the dynamic model of the thrust-vectoring mechanism based on the 3RPS parallel manipulator is established. The schematic diagram of the dynamic analysis model of the 3RPS parallel manipulator is represented graphically, as shown in Figure 12.

Referring to Figure 12, dynamic formulation of the force and moment balances on the linear actuator of the 3RPS parallel manipulator can be expressed as follows:

$$
\left\{\begin{array}{l}
F_{B i}+F_{g i}-F_{b i}-F_{C i}=m_{t} \dot{v}_{t i}, \\
M_{B i}-M_{g i}-M_{C i}+M_{b i}-m_{t} r_{t i} \times \dot{v}_{t i}=I_{i} \dot{\omega}_{i}+\omega_{i} \times I_{i} \omega_{i},
\end{array}\right.
$$




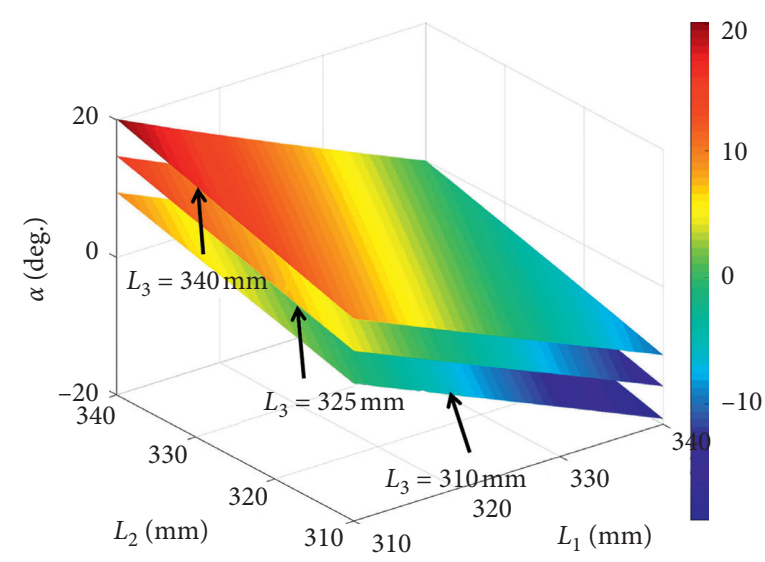

(a)

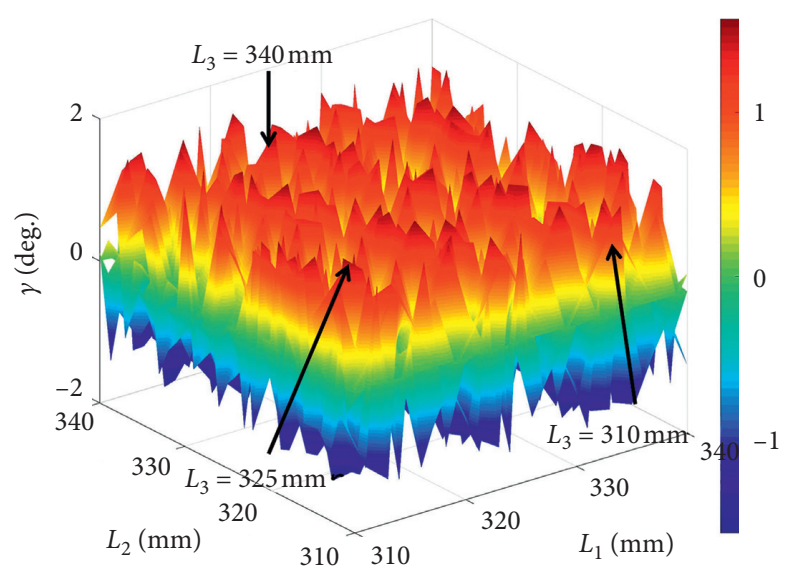

(c)

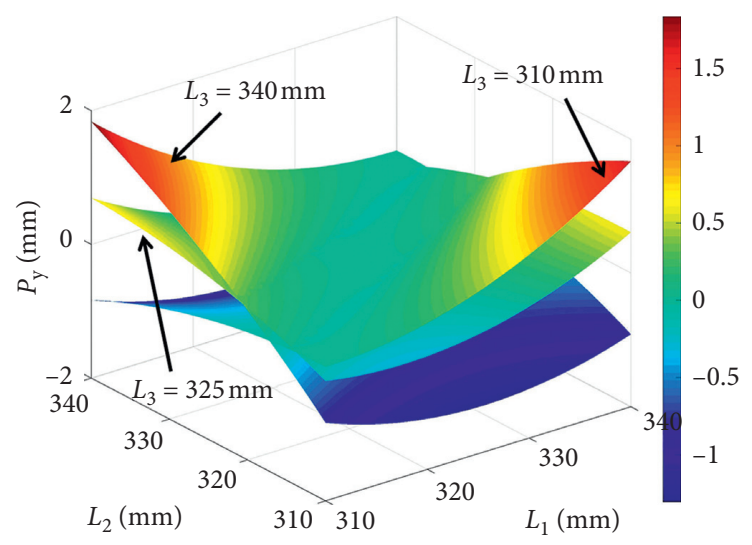

(e)

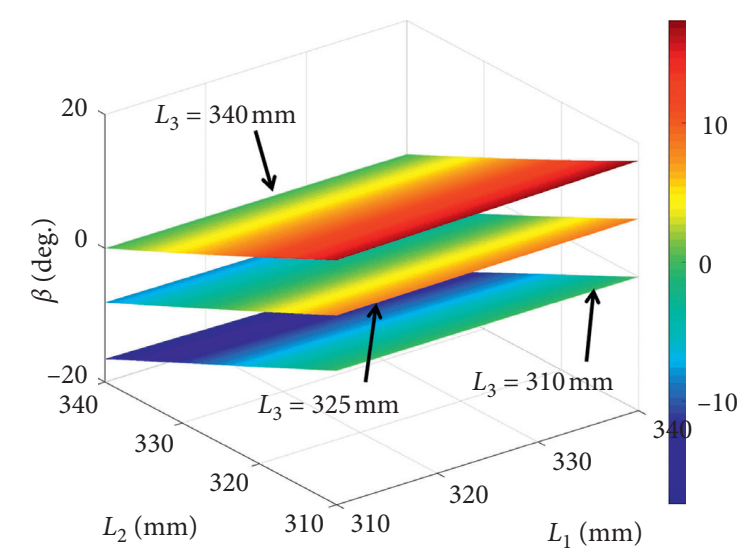

(b)

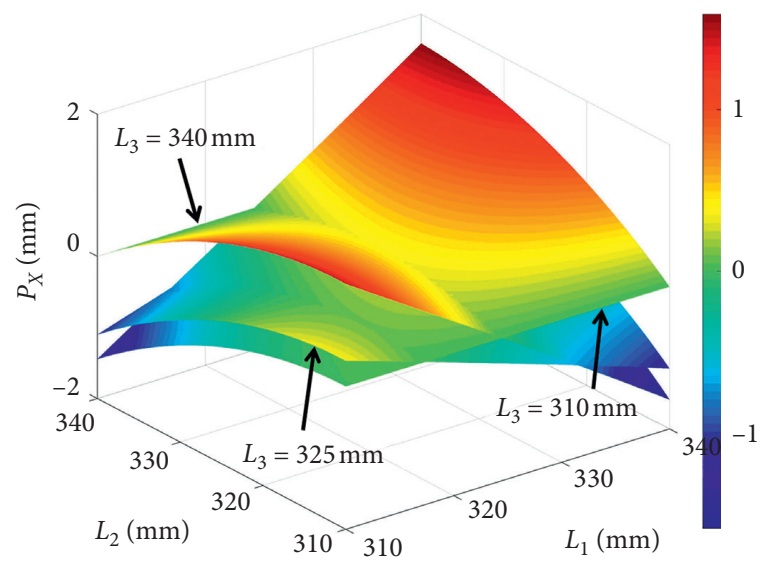

(d)

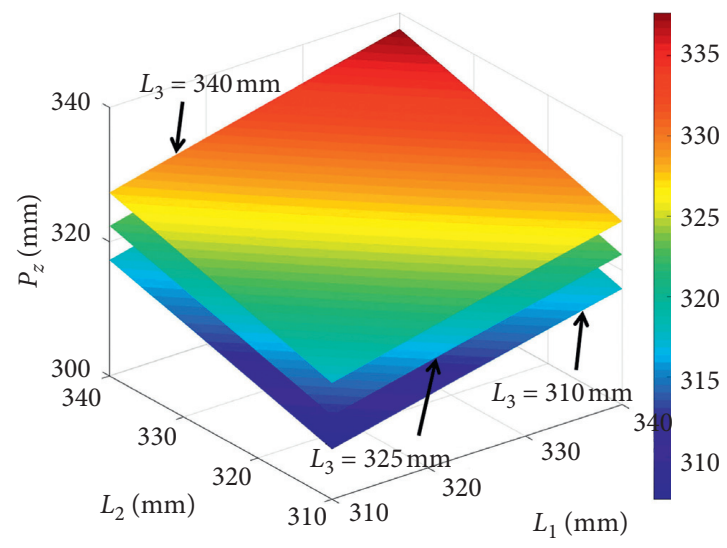

(f)

FIgURE 8: The position and orientation of the top rotating platform with certain actuator lengths.

where $F_{B i}$ and $M_{B i}$ denote force and moment applied at point $B_{i}$, and $F_{C i}$ and $M_{C i}$ denote force and moment at point $C_{i}$ accordingly. $F_{g i}$ and $F_{b i}$ are the gravity and buoyancy of the linear actuator, $M_{g i}$ and $M_{b i}$ denote the moments generated by gravity and buoyancy of the linear actuator, respectively. It should be noted that the buoyancy of the linear actuator $F_{b i}$ can be obtained by the diameters $d_{r}$ and $d_{t}$ of bottom section and the movable part and the length of bottom section $l_{r} . m_{t}$ and $I_{i}$ represent the mass of the translating component and the inertia moment of the linear actuator. $\dot{v}_{t i}$ represents the acceleration velocity of the linear actuator. $r_{t i}$ denotes the vector from point $B_{i}$ to the mass center of linear actuator, and $\dot{\omega}_{i}$ denote the angular velocity and acceleration of the linear actuator, respectively.

Due to acting by an external force and moment, it is necessary to carrying out dynamic analysis of the top moving platform for establishing overall dynamics model for the 


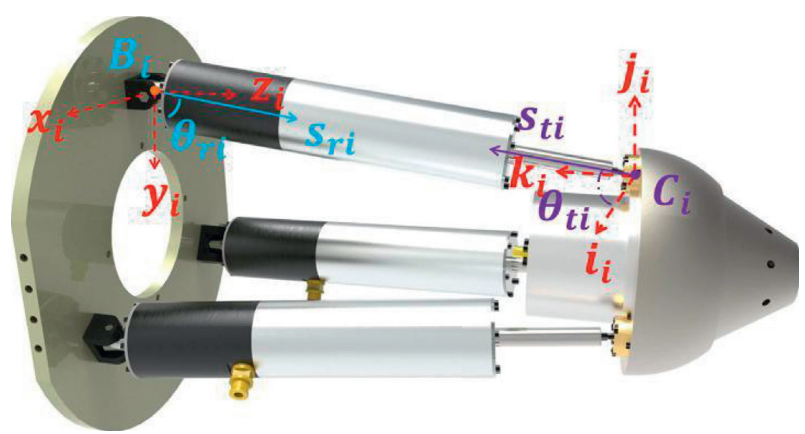

FIGURE 9: Schematic diagram of revolute joint and spherical joint.

thrust-vectoring mechanism. According to the forces and moments distributions analysis of the proposed thrustvectoring mechanism, the stress conditions of the top platform can be represented as Figure 13.

As shown in Figure 13, forces and moments are applied on the connection points $C_{i}$ generated from many respects, such as the linear actuator, the top rotating platform, and duct propeller. Based on the definition of forces and moments at point $C_{i}$ above, the dynamical equations of the top rotating platform can be expressed as

$$
\left\{\begin{array}{l}
\sum_{i=1}^{3} F_{C i}+F_{g-P}-F_{b-P}+R F_{e}=m_{P} \dot{v}_{P}, \\
\sum_{i=1}^{3}\left(M_{C i}\right)-l_{g-P} \times F_{g-P}+l_{b-P} \times F_{b-P}+R M_{e}=I_{P} \dot{\omega}_{P}+\omega_{P} \times\left(I_{P} \omega_{P}\right),
\end{array}\right.
$$

where $v_{p}$ and $\dot{v}_{P}$ are the velocity and acceleration and $\omega_{P}$ and $\dot{\omega}_{P}$ denote the angular velocity and angular acceleration at the center of the top platform, respectively. $F_{C i}$ denotes actuating force from the linear actuator along the direction of actuator. Because the top platform is an axisymmetric structure, $F_{g-P}$ and $F_{b-P}$ are the gravity and buoyancy from the top platform, and $l_{g-P}$ and $l_{b-P}$ denote the distance from the center point $P$ generated by connection points $C_{1} C_{2} C_{3}$ to the center of mass and buoyancy of the top rotating platform, respectively. $F_{e}$ and $M_{e}$ are the external force and moment mainly generated from the propeller and the duct in our paper. Referring to Figure 13, the external force $F_{e}$ and external moment $M_{e}$ can be defined as

$$
\left\{\begin{array}{l}
F_{e}=F_{\text {prop }}+F_{\text {duct }}, \\
M_{e}=M_{\text {duct }}
\end{array}\right.
$$

where $F_{\text {prop }}$ and $F_{\text {duct }}$ denote the force generated by the propeller and the duct of the thrust-vectoring mechanism and $M_{e}$ denotes the moment generated by the duct, respectively. The thrust vector $F_{\text {prop }}$ is produced by the propeller and can be expressed as

$$
F_{\text {prop }}=T_{p}\left[\begin{array}{c}
-c \alpha c \beta \\
c \alpha s \beta \\
s \alpha
\end{array}\right]
$$

where $T_{p}$ denotes the thrust produced by propeller and based on standard propeller theory $[28,31], T_{p}=K_{T} \rho n^{2}{ }_{p} D^{4} . K_{T}, \rho$, $n_{p}$, and $D$ denote the thrust coefficient, the water density, the rotation speed of the propeller, and the propeller diameter, respectively.

In this AUV, the duct propeller has been widely adopted to protect from damage and improve the propulsive efficiency by being enclosed by a duct. To further investigate the dynamic model of the vectored thruster, it is clearly necessary to considerate the effect on lift and drag generated by the duct. The force generated by the duct applied to the platform can be expressed as

$$
F_{L D}=\left[\begin{array}{c}
0 \\
-L \\
D
\end{array}\right] \text {, }
$$

where $L$ and $D$ denote the lift and drag of the duct, which can be calculated by CFD software.

Because the duct rotates around the center of the duct in use, a transformation matrix $R_{d}$ is established to convert the duct frame into the body frame, and the matrix $R_{d}$ can be described as

$$
R_{d}=\left[\begin{array}{ccc}
c \alpha c \beta & -s \alpha & c \alpha s \beta \\
s \alpha c \beta & c \alpha & s \alpha s \beta \\
-s \beta & 0 & c \beta
\end{array}\right]
$$

where $\alpha$ and $\beta$ represent the tilt angles of the ducted propeller.

Referring to equations (17)-(20), the force $F_{\text {duct }}$ and moment $M_{\text {duct }}$ generated by the duct that are applied on the platform of the thrust-vectoring mechanism can be calculated as

$$
\left\{\begin{array}{l}
F_{\text {duct }}=R_{d} F_{L D} \\
M_{\text {duct }}=r_{p} \times F_{\text {duct }} .
\end{array}\right.
$$

Finally, based on the above analysis and according to Figures 12 and (15)-(21), the force balance along the leg direction can be expressed as

$$
F_{i}=F_{B i} \cdot s_{i},
$$

where $F_{i}$ denotes the force produced by the linear actuator to complete the key components of drive function and $s_{i}$ is the unit vector of the $i$ th actuator.

In order to do a better research on the effect of motion on the vectored thruster, the numerical dynamic simulation on the thrust-vectoring mechanism has been developing. Some parameters used in the simulation, such as the dimension parameters of vectored thruster, are calculated through CAD software, and other parameters can be obtained by in [56]. All parameters of the thrust-vectoring mechanism are given in Table 2.

Based on the abovementioned theory analysis and parameters, the analysis formulations in Section 4 have been implemented in MATLAB.

When only considering the thrust produced by the duct propeller and the top platform moving according to $\alpha=\alpha_{s}$ and $\beta=0$, the length changes and the driving forces of linear 


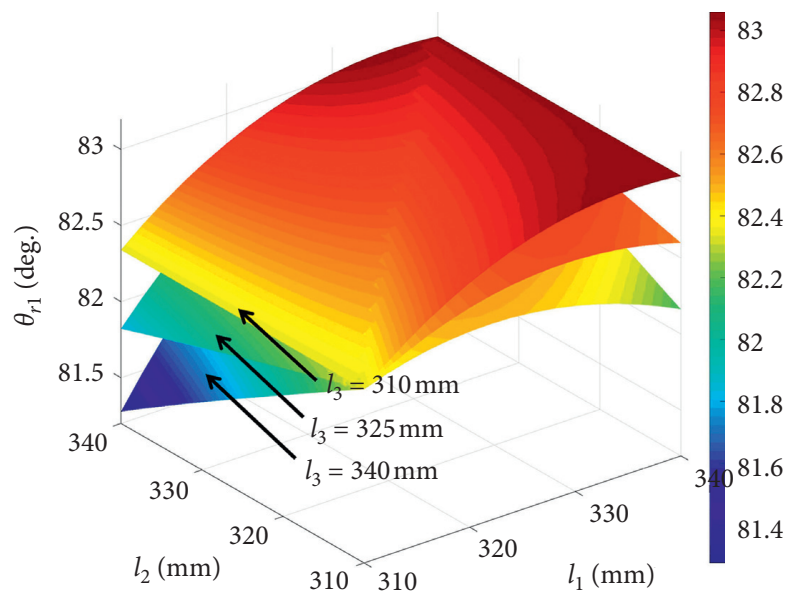

(a)

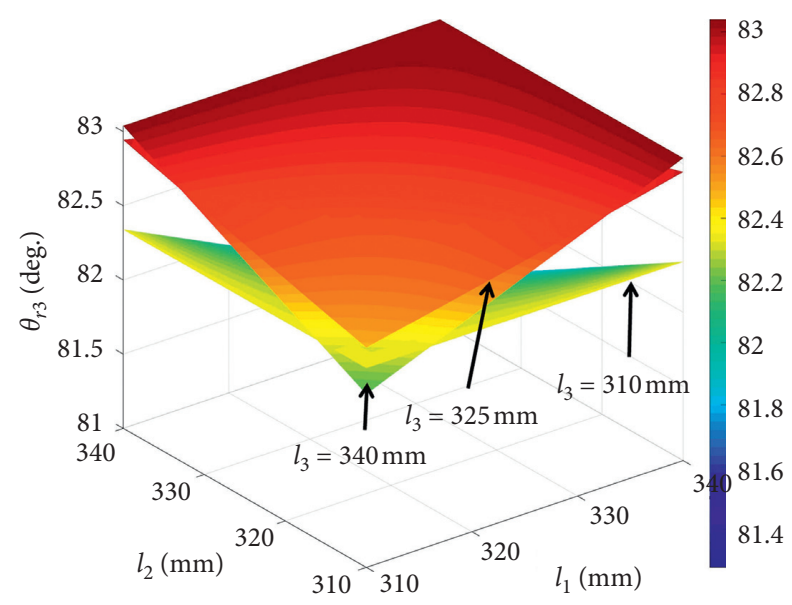

(c)

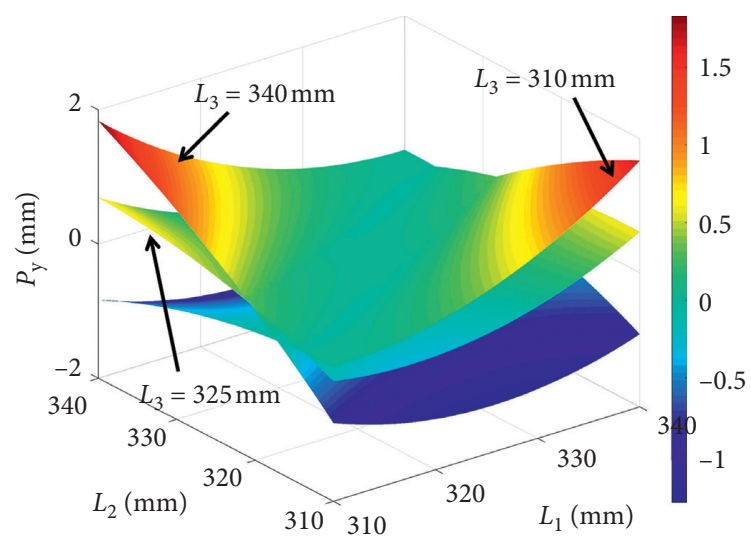

(e)

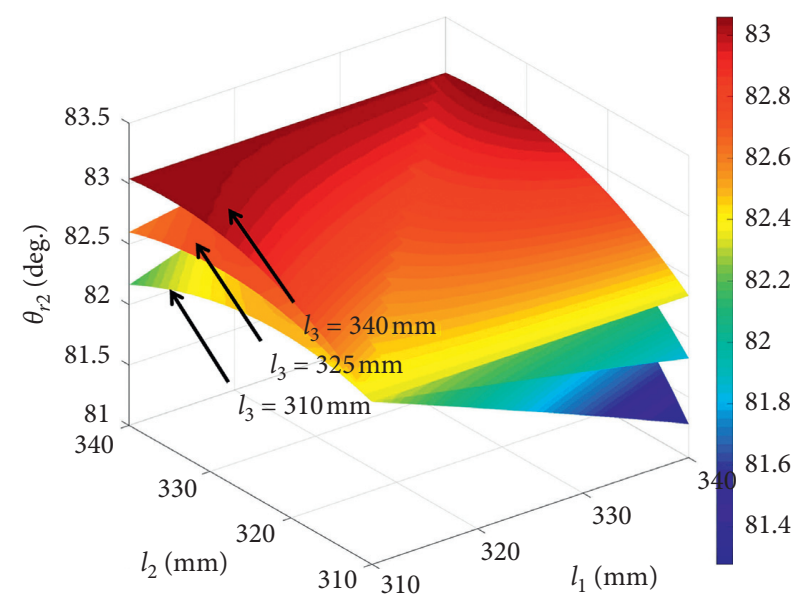

(b)

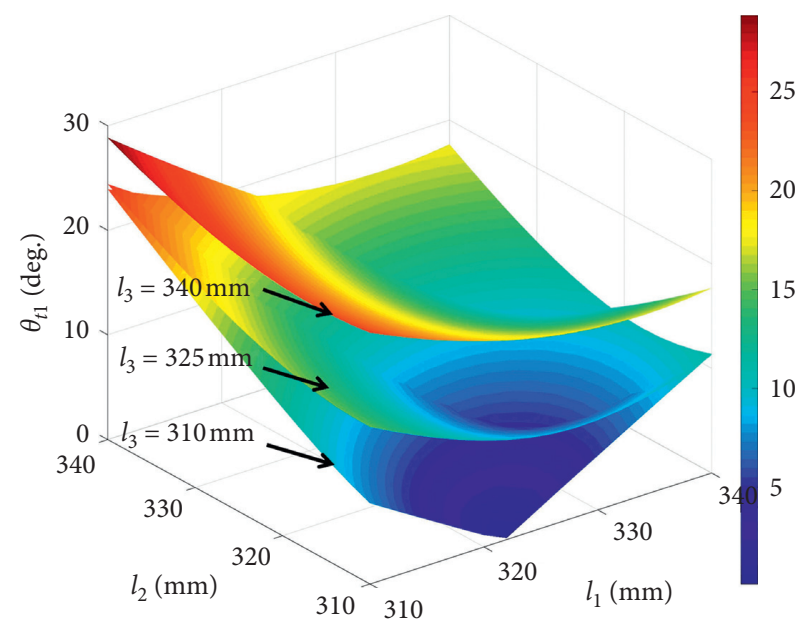

(d)

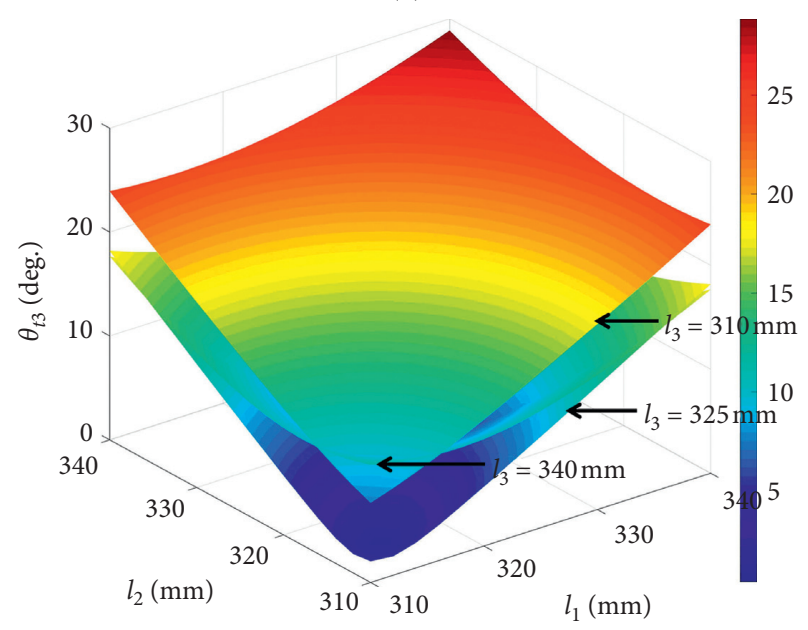

(f)

FIGURE 10: Tilt angle of revolute and spherical joint of the actuator by forward kinematic analysis.

actuator can be calculated, and the results are plotted in Figures 14 and 15.

In the dynamic analysis above, the gravity, buoyancy, drag, and torque of the vectored thruster is ignored. When the weights of the rotating platform and the three actuators are taken into account only, the driving forces of the linear actuator can be calculated and plotted in Figure 16.

In addition, the buoyancy of actuator depends on the length changes of actuators, and the buoyancy of linear actuator can be calculated by the length change. When the 

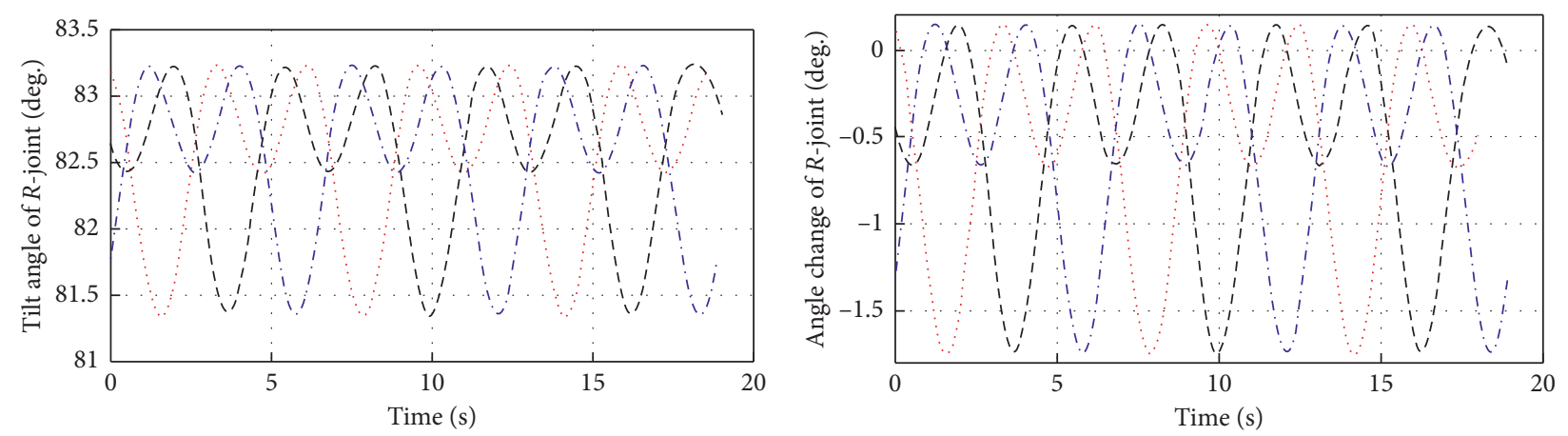

$$
\begin{aligned}
& \ldots \theta_{r 1} \\
& \ldots-\theta_{r 2} \\
& \ldots-\theta_{r 3}
\end{aligned}
$$

(a)

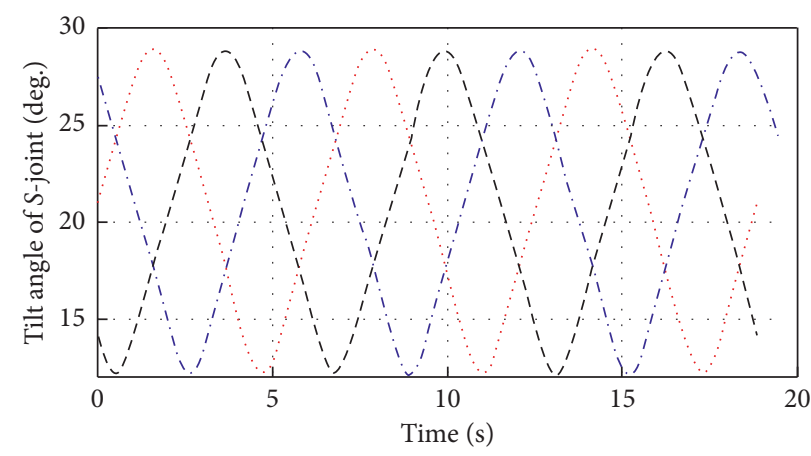

$$
\begin{gathered}
\ldots \theta_{t 1} \\
\cdots-\theta_{t 2} \\
---\theta_{t 3}
\end{gathered}
$$

(c)

$$
\begin{aligned}
& \ldots . . \Delta \theta_{r 1} \\
& \ldots-\Delta \theta_{r 2} \\
& ---\Delta \theta_{r 3}
\end{aligned}
$$

(b)

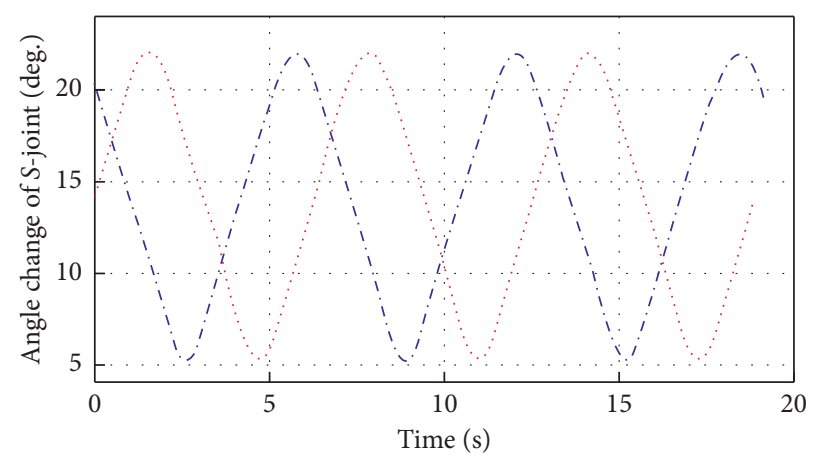

$$
\begin{aligned}
& \ldots . . \Delta \theta_{t 1} \\
& \ldots-\Delta \theta_{t 2} \\
& \ldots--\Delta \theta_{t 3}
\end{aligned}
$$

(d)

FIGURE 11: The tilt angle and angle change. (a) $\theta_{r i}$. (b) $\Delta \theta_{r i}$. (c) $\theta_{t i}$. (d) $\Delta \theta_{t i}$.

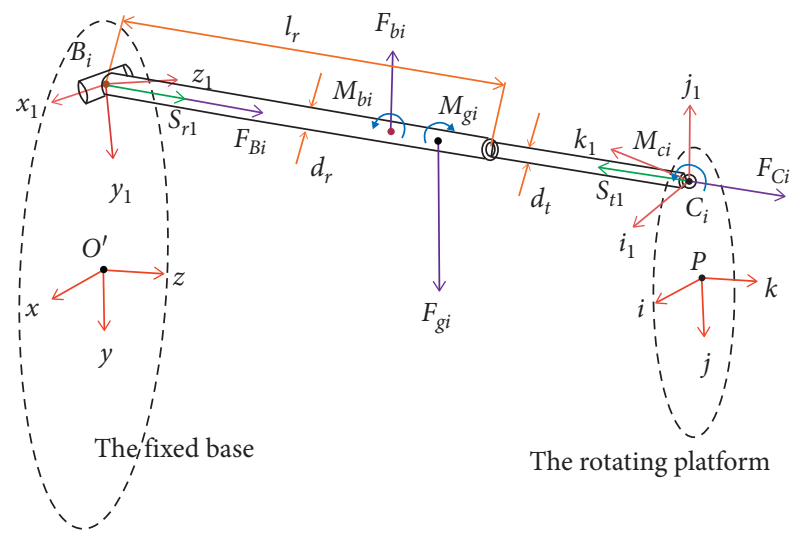

Figure 12: Schematic diagram of the dynamic model of the thrustvectoring mechanism.

top platform moves according to the designed trajectory, the buoyancy is created with the movement of the vectored thruster and the result of buoyancy is shown in Figure 17.

As we can see in Figure 17, the buoyant forces of actuators change with the length changes of actuators, but the

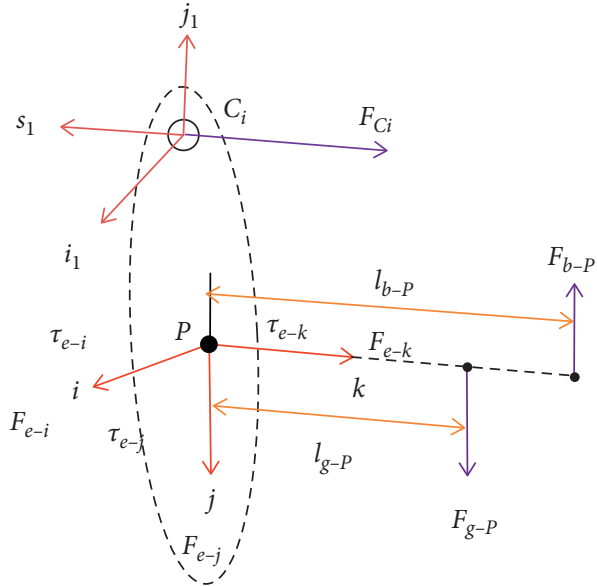

The top platform

FIgURE 13: Schematic diagram of the dynamic analysis of the rotating platform.

magnitude of the change is relatively small. Hence, the buoyant forces of actuators can be approximately equal to $4.7 \mathrm{~N}$ in this next calculation. 
TABle 2: Parameters of the thrust-vectoring mechanism.

\begin{tabular}{lc}
\hline Symbol & Value (unit) \\
\hline$d_{r}$ & $50(\mathrm{~mm})$ \\
$d_{\mathrm{t}}$ & $10(\mathrm{~mm})$ \\
$l_{\mathrm{r}}$ & $250(\mathrm{~mm})$ \\
$l_{g-P}$ & $35(\mathrm{~mm})$ \\
$l_{b-p}$ & $70(\mathrm{~mm})$ \\
$F_{g-P}$ & $100(\mathrm{~N})$ \\
$F_{b-p}$ & $30(\mathrm{~N})$ \\
$F_{g i}$ & $20(\mathrm{~N})$ \\
$T_{p}$ & $200(\mathrm{~N})$ \\
$I_{p}$ & $\operatorname{diag}(3.21 .54)\left(\mathrm{kg} \cdot \mathrm{m}^{2}\right)$ \\
$I_{i}$ & $\operatorname{diag}(24.524 .50 .1)\left(\mathrm{kg} \cdot \mathrm{m}^{2}\right)$ \\
$m_{t}$ & $0.1(\mathrm{~kg})$ \\
$P$ & 60000 \\
$I$ & 40000 \\
$D$ & 15000 \\
\hline
\end{tabular}

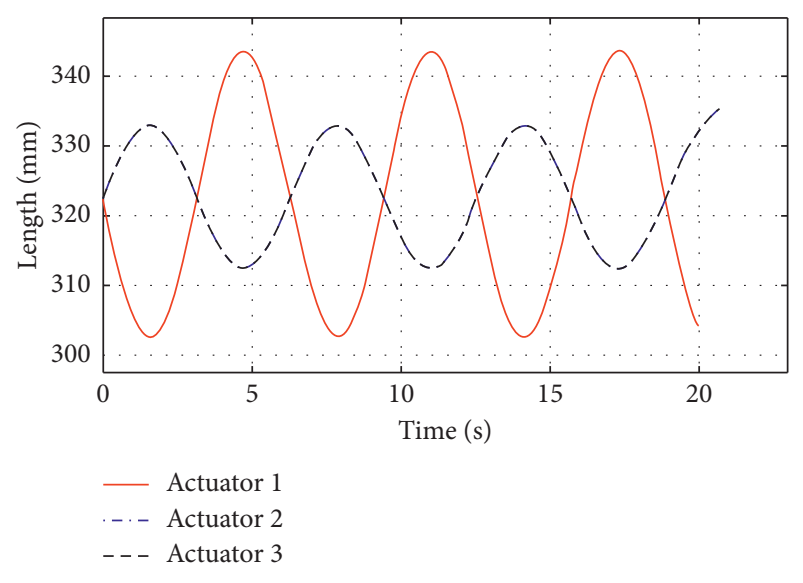

FIgURE 14: Length change of actuators at $\alpha=\alpha_{s}$ and $\beta=0$.

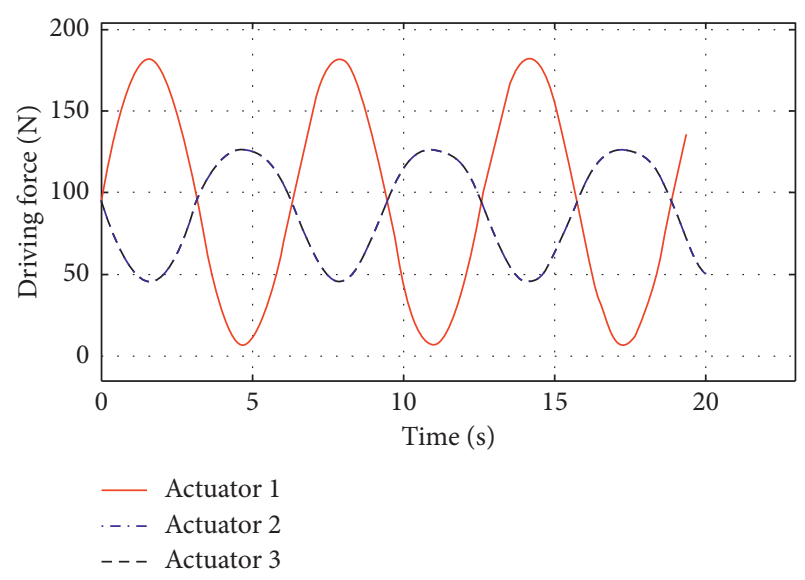

FIgURE 15: Driving forces of actuators at $\alpha=\alpha_{s}$ and $\beta=0$.

Generally, the emphases of dynamic analysis of the parallel manipulator for research are mainly focused on the gravity, the external force, and torques. However, the buoyancy is also an important factor that affects the driving force of the actuators because the designed vectored thruster is used in underwater vehicles. In addition,

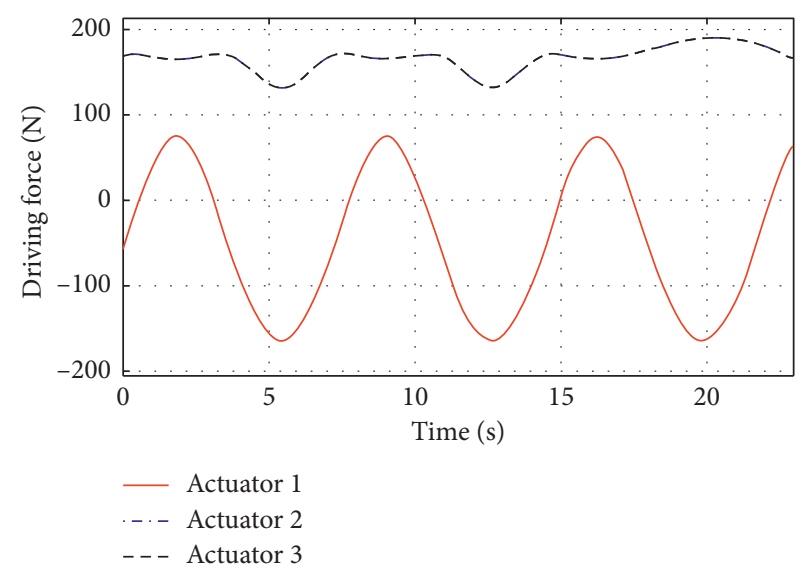

FIgURE 16: Driving forces of actuators at $\alpha=\alpha_{s}$ and $\beta=0$.

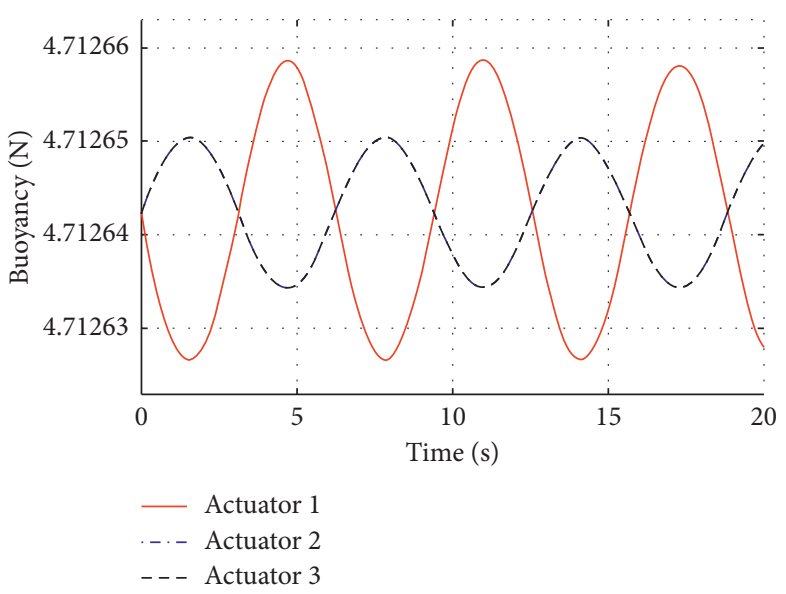

Figure 17: Buoyant forces of actuators at $\alpha=\alpha_{s}$ and $\beta=0$.

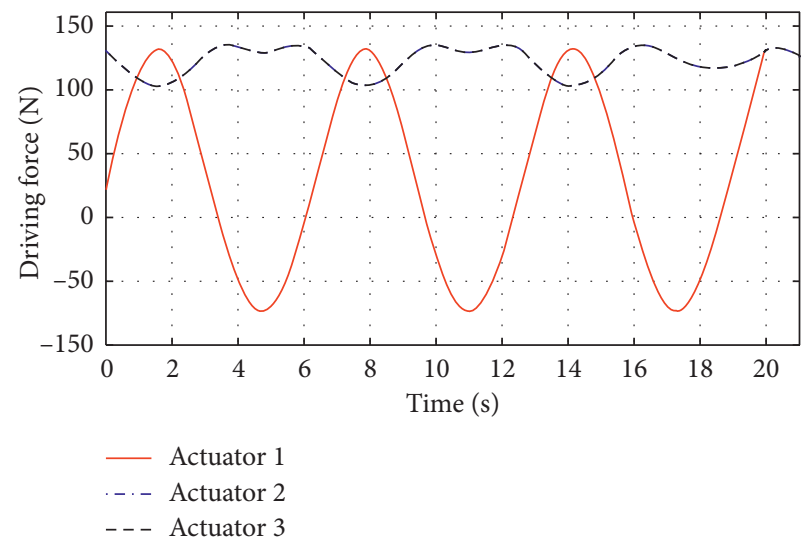

FIgURE 18: Driving forces of actuators at $\alpha=\alpha_{s}$ and $\beta=0$.

the buoyancy of the other parts can be directly calculated by CAD software.

With considering the buoyant forces of actuators as shown in Figure 17 and buoyancy of other parts, the driving force of the $i$ th actuator can be recalculated and plotted in Figure 18. 


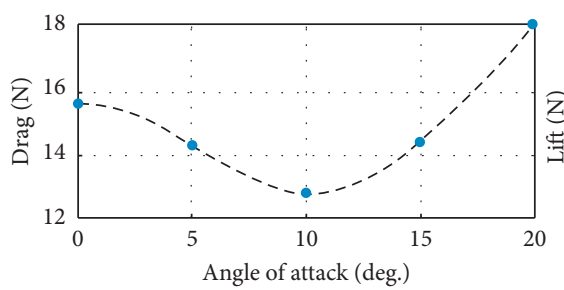

(a)

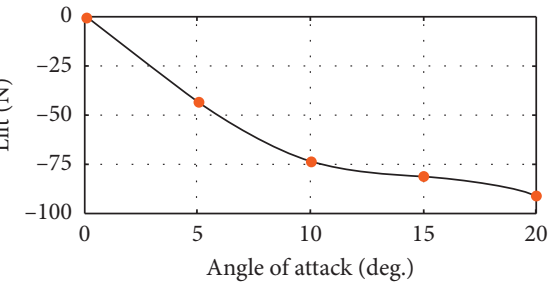

(b)

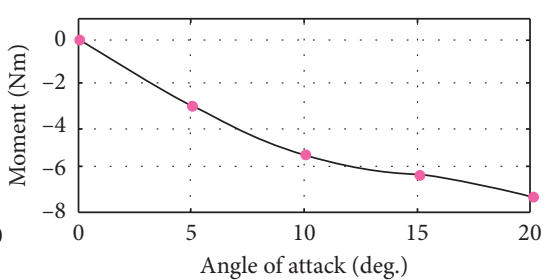

(c)

Figure 19: Drag (a), lift (b), and pitch moment (c) with different angles of attack.

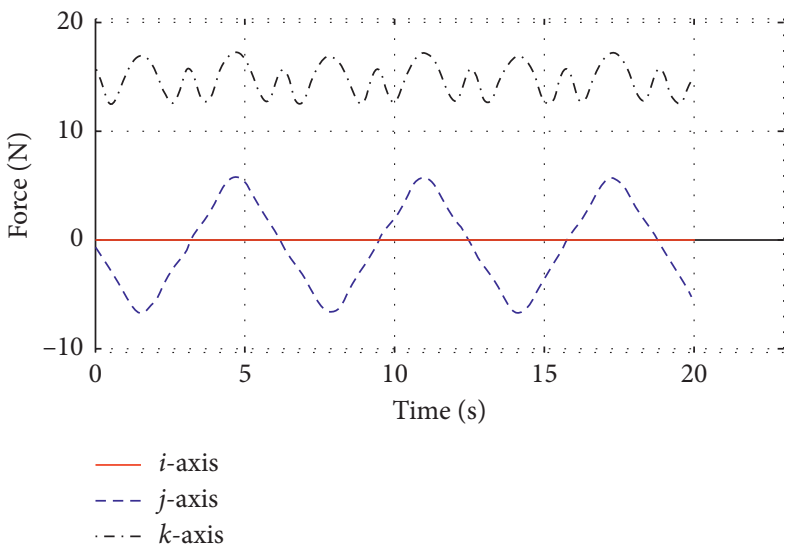

(a)

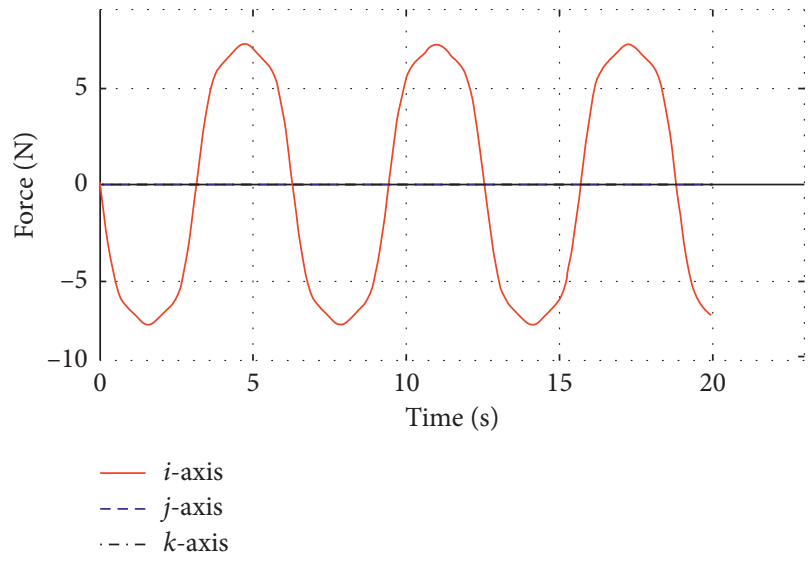

(b)

Figure 20: Force (a) and moment (b) generated by the duct at $\alpha=\alpha_{s}$ and $\beta=0$.

Comparing the driving forces in Figures 15, 17, and 18, it is obvious that the gravity and buoyancy of the whole vectored thruster have an effect on the value of the driving force greatly.

In order to investigate the performance and characteristic of the vectored thruster more fully, some influential factors of the duct have been considered in the following simulations. Referring to (19)-(21), the parameters of the duct are important to calculate in the numerical dynamic simulation. In this paper, we use CFD simulation to get the dynamics parameters of the duct. Figure 19 shows the drag, lift, and moment of the duct with the angle of attack range between $0^{\circ}$ and $20^{\circ}$ and maintains the flow velocity at $2.5 \mathrm{~m} / \mathrm{s}$, respectively.

Based on the dynamics parameters of the duct, as shown in Figure 19, the force and moment generated by the duct are calculated and presented in Figure 20.

The calculation results show that the influential factors of the duct play a very important role in calculating the driving forces of actuators; hence, the influential factors of the duct should be considered in a calculation schedule.

To improve the performance of the vehicle motion control, the tilt angles $\alpha$ and $\beta$ play a crucial role in the vectored thruster AUVS. With considering the usage of the vectored thruster, a control scheme using the PID method is developed for the designed thrust-vectoring mechanism, as shown in Figure 21. PID algorithm is the most widely used control methods in all kinds of application fields for its effectiveness and practicability. Based on the control scheme shown in Figure 21, a control model of the proposed thrustvectoring mechanism is developed by Matlab and Simscape Mutibody, as shown in Figures 22 and 23.

To further investigate the performance of proposed control model, related numerical simulations are carried out with top platform moves according to $=\alpha_{s}$ and $\beta=0$. The related parameters are given in Table 2, the length responses of linear actuators can be obtained, as shown in Figure 24.

The simulation results from Figures 14 and 24 show that the designed PID controller for the thrust-vectoring mechanism is fast, effective, and able to achieve the expected goal commendably. Based on the designed controller and considering the influence of factors as analyzed above, which includes extra forces and moments from duct and buoyant forces of the vectored thruster, the driving force of linear actuators is presented in Figure 25.

Comparing the driving force in Figures 18 and 25, the driving forces of actuators have some similarities in change trend at the same time, but the maximum and minimum of driving forces are distinctly different. Hence, it is concluded that the lift, drag, and torque of the duct propeller are important influential factors to the dynamic model of the vectored thruster. Accordingly, for the purposes of optimizing the structure and decreasing the dimension, it is of significance to choose the appropriate linear actuator for driving the proposed thrust-vectoring mechanism by studying the dynamic analysis with considering all kinds of influence factor. 


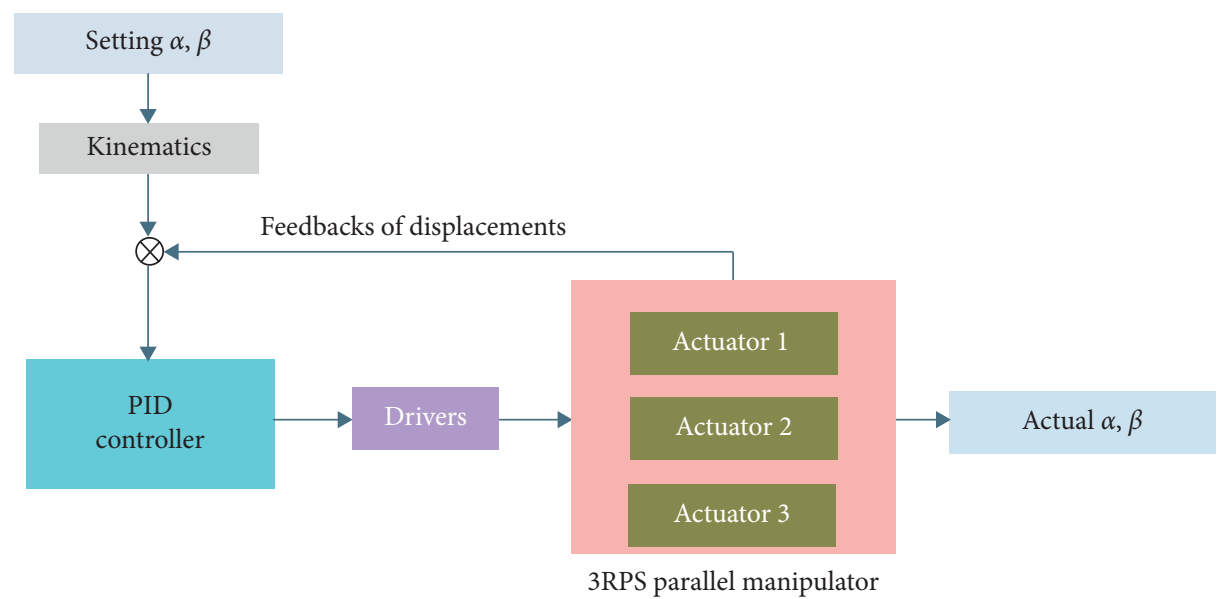

FIgURE 21: Control scheme of the thrust vector control system.

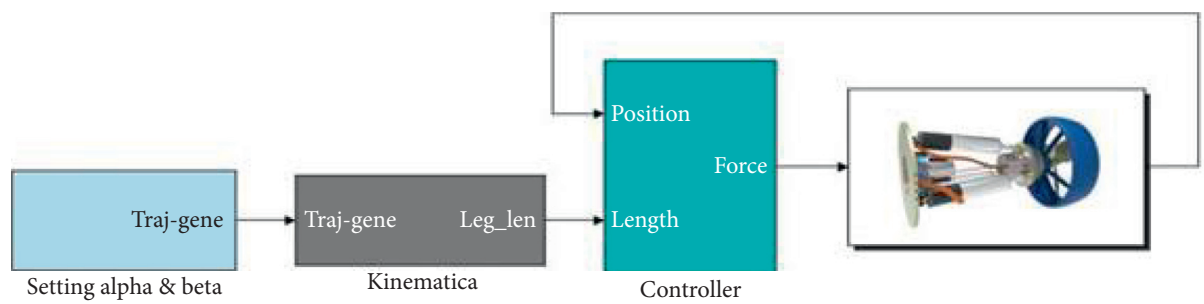

Figure 22: Control scheme of the thrust-vectoring mechanism.

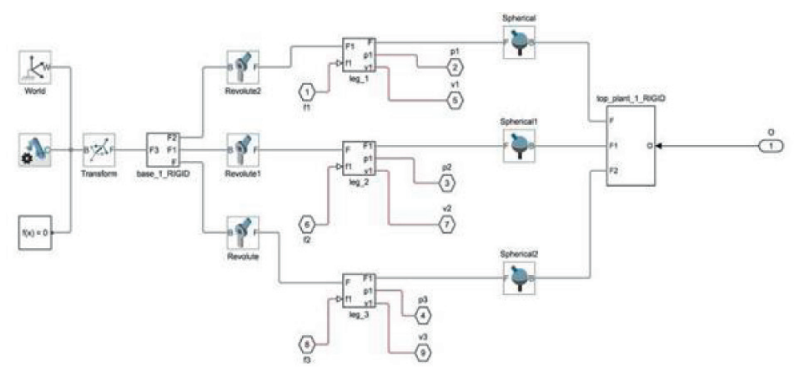

FIGURE 23: Model of the the thrust-vectoring mechanism with Simscape Mutibody.

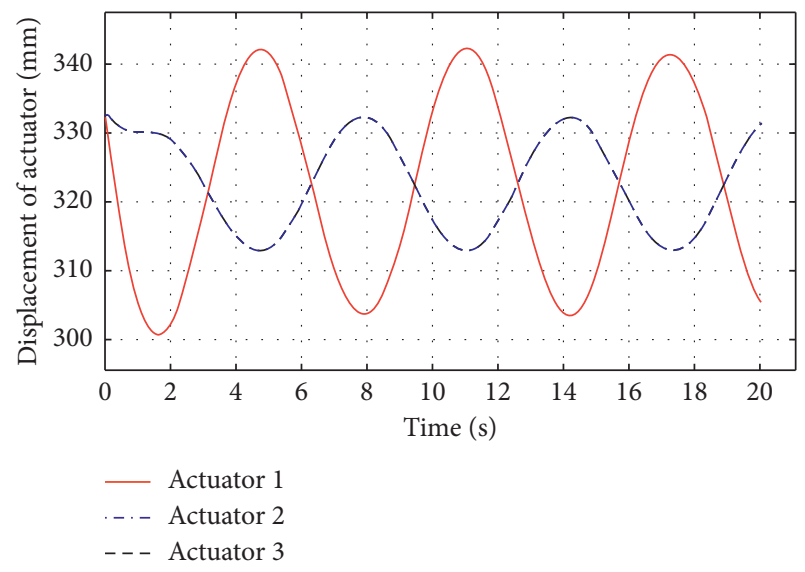

Figure 24: The length response of the linear actuator.

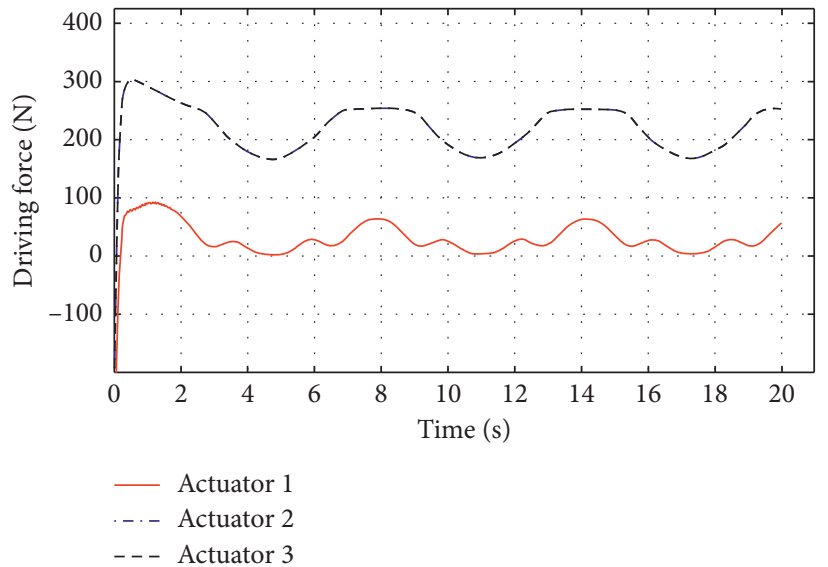

FIGURE 25: Driving forces of linear actuator.

\section{Conclusion}

In this paper, a design scheme for the vectored thruster based on a 3RPS parallel manipulator is proposed to solve the effect of the control surface weakening problems. Parallel manipulators have several advantages over the mechanical structure and are suitable for various application fields, such as compact and reasonable structure, fast response, and high positioning precision. Because of the merits of itself, this type mechanical structure is used to design the thrustvectoring mechanism considering the limited space and hash environment. Additionally, a duct propeller is adopted 
as power source of the proposed vectored thruster, which is installed on the top rotating platform with a main motor as a whole structure, thereby this design ensures compact structure, reliable motion, and high propulsive efficiency. Because the control force is provided by the component force of thrust rather than common rudders, the proposed vectored thruster AUVs have the abilities to complete all kinds of certain tasks and operations at a low forward speed.

In order to make sure the designed vectored thruster can run efficiently and stably, studying and developing the control system is fundamental to implement the design function of the vectored thruster. Owing to the importance of control system, related theoretical research about kinematics and dynamics of the thrust-vectoring mechanism is carried out to establish the motion model. In the kinematic analysis, the inverse and forward kinematics of the thrustvectoring mechanism is presented, and the numerical simulations are accomplished to prove the feasibility and effectiveness of this design. In the section of workspace analysis, the study of the tilt angles of revolute and spherical joints is also carried out to make sure the motion platform can implement its designed function in limited motion space. In order to further verify feasibility of the thrustvectoring mechanism, based on the considerations of various affecting factors, a dynamics model of the designed thrust-vectoring mechanism is established according to theoretical analysis, and the driving forces of the linear actuator are presented though a series of numerical simulations. In addition, a control scheme based on PID algorithm is proposed for the thrust vector control system on the existing work basis, and a control model is established using Simsacpe Mutibody, and the simulation results proved the feasibility of the proposed control scheme, which can effectively realize the goal of controlling the thrust-vectoring mechanism.

According to the above, the designed vectored thruster is able to provide the vectored thrust effectively and efficiently, and the AUVs equipped with vectored thruster are able to complete a variety of the complex tasks at a comparably low forward speed.

In the future research, a series of numerical simulations and theoretical study are carried out to investigate hydrodynamic performance of this vectored thruster AUV. On this basis, a prototype of this designed vectored thruster will be developed and experimental test will be carried out to verify the principles design. Moreover, the corresponding control system of the vectored thruster as a part of the AUV will be developed and tested in pools or open water to check its performance.

\section{Data Availability}

The data used to support the findings of this study are included within the article.

\section{Conflicts of Interest}

The authors declare that they have no conflicts of interest.

\section{Acknowledgments}

This work was supported by the National Natural Science Foundation of China (Grant no. 51879220).

\section{References}

[1] V. Upadhyay, S. Gupta, A. C. Dubey et al., "Design and motion control of autonomous underwater vehicle," in Proceedings of the 2015 IEEE Underwater Technology (UT), Chennai, India, February 2015.

[2] J. Mccolgan and E. Mcgookin, "Coordination of multiple biomimetic autonomous underwater vehicles using strategies based on the schooling behaviour of fish," Robotics, vol. 5, no. 1, p. 2, 2016.

[3] M. Furlong, D. Paxton, P. Stevenson, M. Pebody, S. D. Mcphail, and J. Perrett, "Autosub long range: a long range deep diving AUV for ocean monitoring," in Proceedings of the 2012 IEEE/OES Autonomous Underwater Vehicles $(A U V)$, Southampton, UK, September 2012.

[4] B.-H. Jun, J.-Y. Park, F.-Y. Lee et al., "Development of the AUV "ISiMI" and a free running test in an ocean engineering basin," Ocean Engineering, vol. 36, no. 1, pp. 2-14, 2009.

[5] Y. Zhang, Y. Li, Y. Sun, J. Zeng, and L. Wan, "Design and simulation of X-rudder AUV'S motion control," Ocean Engineering, vol. 137, pp. 204-214, 2017.

[6] S. K. Jain, S. Mohammad, S. Bora, and M. Singh, "A review paper on: autonomous underwater vehicle," International Journal of Scientific \& Engineering Research, vol. 6, no. 2, 2015.

[7] J. Elvander and G. Hawkes, "ROVs and AUVs in support of marine renewable technologies," in Proceedings of the 2012 Oceans, Hampton Roads, VA, USA, October 2012.

[8] Y. Allard and E. Shahbazian, "Unmanned underwater vehicle (UUV) information study," Technical report, OODA Technologies Inc, Montreal, Canada, 2014.

[9] Y. Wang, B. Jiang, Z.-G. Wu, S. Xie, and Y. Peng, "Adaptive sliding mode fault-tolerant fuzzy tracking control with application to unmanned marine vehicles," IEEE Transactions on Systems, Man, and Cybernetics: Systems, pp. 1-10, 2020.

[10] T. I. Fossen and O.-E. Fjellstad, "Robust adaptive control of underwater vehicles: a comparative study," IFAC Proceedings Volumes, vol. 28, no. 2, pp. 66-74, 1995.

[11] Y. Wang, C. K. Ahn, H. Yan, and S. Xie, "Fuzzy control and filtering for nonlinear singularly perturbed Markov jump systems," IEEE Transactions on Cybernetics, pp. 1-12, 2020.

[12] Y. Wang, H. R. Karimi, H.-K. Lam, and H. Yan, "Fuzzy output tracking control and filtering for nonlinear discrete-time descriptor systems under unreliable communication links," IEEE Transactions on Cybernetics, vol. 50, no. 6, pp. 23692379, 2019.

[13] Y. Wang, X. Xie, M. Chadli, S. Xie, and Y. Peng, "Sliding mode control of fuzzy singularly perturbed descriptor systems," IEEE Transactions on Fuzzy Systems, p. 1, 2020.

[14] T. Ura, T. Obara, S. Takagawa, and T. Gamo, "Exploration of Teisi knoll by autonomous underwater vehicle R-one robot," in Proceedings of the MTS/IEEE Oceans 2001. An Ocean Odyssey, Honolulu, HI, USA, November 2001.

[15] P. B. Sujit, J. B. De Sousa, and F. L. Pereira, "UAV and AUVS coordination for ocean exploration," in Proceedings of the OCEANS 2009-EUROPE, Bremen, Germany, May 2009.

[16] K. Weitemeyer and S. Constable, "Mapping shallow geology and gas hydrate with marine CSEM surveys," First Break, vol. 28, no. 6, 2010. 
[17] P. Wessel and M. T. Chandler, "The spatial and temporal distribution of marine geophysical surveys," Acta Geophysica, vol. 59, no. 1, pp. 55-71, 2011.

[18] M. Grasmueck, G. P. Eberli, D. A. Viggiano, T. Correa, G. Rathwell, and J. Luo, "Autonomous underwater vehicle (AUV) mapping reveals coral mound distribution, morphology, and oceanography in deep water of the straits of Florida," Geophysical Research Letters, vol. 33, no. 23, 2006.

[19] S. A. T. Randeni, A. Forrest, R. Cossu, Z. Leong, D. Ranmuthugala, and V. Schmidt, "Parameter identification of a nonlinear model: replicating the motion response of an autonomous underwater vehicle for dynamic environments," Nonlinear Dynamics, vol. 91, no. 2, pp. 1229-1247, 2018.

[20] M. D. Henschel, R. Olsen, P. Hoyt, and P. W. Vachon, "The ocean monitoring workstation: experience gained with radarsat," in Proceedings of the 1997 Geomatics in the ERA of RADARSAT (GER'97), Ottawa, Canada, 1997.

[21] D. C. Webb, P. J. Simonetti, and C. P. Jones, "SLOCUM: an underwater glider propelled by environmental energy," IEEE Journal of Oceanic Engineering, vol. 26, no. 4, pp. 447-452, 2001.

[22] L. A. Henkel, H. Nevins, M. Martin, S. Sugarman, J. T. Harvey, and M. H. Ziccardi, "Chronic oiling of marine birds in California by natural petroleum seeps, shipwrecks, and other sources," Marine Pollution Bulletin, vol. 79, no. 1-2, pp. 155-163, 2014.

[23] N. Gracias, P. Ridao, R. Garcia et al., "Mapping the Moon: using a lightweight AUV to survey the site of the 17th century ship La Lune," in Proceedings of the OCEANS-Bergen 2013, Bergen, Norway, June 2013.

[24] K. Kornei, "Seafloor data from lost airliner search are publicly released," EOS, vol. 98, 2017.

[25] M. Purcell, D. Gallo, G. Packard et al., "Use of REMUS 6000 AUVs in the search for the air France flight 447," in Proceedings of the OCEANS 2011, Waikoloa, HI, USA, September 2011.

[26] M. H. Khodayari and S. Balochian, "Modeling and control of autonomous underwater vehicle (AUV) in heading and depth attitude via self-adaptive fuzzy PID controller," Journal of Marine Science and Technology, vol. 20, no. 3, pp. 559-578, 2015.

[27] D. R. Blidberg, "The development of autonomous underwater vehicles (AUV); a brief summary," IEEE ICRA, vol. 4, p. 1, 2001.

[28] K. Tanakitkorn, P. A. Wilson, S. R. Turnock, and A. B. Phillips, "Depth control for an over-actuated, hover-capable autonomous underwater vehicle with experimental verification," Mechatronics, vol. 41, pp. 67-81, 2017.

[29] B. Xin, L. Xiaohui, S. Zhaocun, and Z. Yuquan, “A vectored water jet propulsion method for autonomous underwater vehicles," Ocean Engineering, vol. 74, pp. 133-140, 2013.

[30] T. Liu, Y. Hu, H. Xu, Z. Zhang, and H. Li, "Investigation of the vectored thruster AUVS based on 3SPS-s parallel manipulator," Applied Ocean Research, vol. 85, pp. 151-161, 2019.

[31] K. Tanakitkorn, P. A. Wilson, S. R. Turnock, and A. B. Phillips, "Sliding mode heading control of an overactuated, hovercapable autonomous underwater vehicle with experimental verification," Journal of Field Robotics, vol. 35, no. 3, pp. 396-415, 2018.

[32] X. Lin and S. Guo, "Development of a spherical underwater robot equipped with multiple vectored water-jet-based thrusters," Journal of Intelligent \& Robotic Systems, vol. 67, no. 3-4, pp. 307-321, 2012.
[33] I. Carlucho, M. De Paula, S. Wang, Y. Petillot, and G. G. Acosta, "Adaptive low-level control of autonomous underwater vehicles using deep reinforcement learning," Robotics and Autonomous Systems, vol. 107, pp. 71-86, 2018.

[34] T. Wang and B.-w. Song, "Control of dynamic position system for AUV with multiple thrusters," Acta Armamentarii, vol. 5, 2006.

[35] T. Liu, Y. Hu, H. Xu, Q. Wang, and W. Du, “A novel vectored thruster based on 3-RPS parallel manipulator for autonomous underwater vehicles," Mechanism and Machine Theory, vol. 133, pp. 646-672, 2019.

[36] R. Panish, "Dynamic control capabilities and developments of the Bluefin robotics AUV fleet," in Proceedings of the 16th International Symposium on Unmanned Untethered Submersible Technology (UUST), Durham, NH, USA, 2009.

[37] L. E. Bird, A. Sherman, and J. Ryan, "Development of an active, large volume, discrete seawater sampler for autonomous underwater vehicles," in Proceedings of the OCEANS 2007, Vancouver, Canada, September 2007.

[38] Y. Zhang, R. S. McEwen, J. P. Ryan et al., "A peak-capture algorithm used on an autonomous underwater vehicle in the 2010 Gulf of Mexico oil spill response scientific survey," Journal of Field Robotics, vol. 28, no. 4, pp. 484-496, 2011.

[39] H. Cheng, Y.-K. Yiu, and Z. Li, "Dynamics and control of redundantly actuated parallel manipulators," IEEE/ASME Transactions on Mechatronics, vol. 8, no. 4, pp. 483-491, 2003.

[40] D. Liang, Y. Song, and T. Sun, "Nonlinear dynamic modeling and performance analysis of a redundantly actuated parallel manipulator with multiple actuation modes based on FMD theory," Nonlinear Dynamics, vol. 89, no. 1, pp. 391-428, 2017.

[41] A. G. Ruiz, J. C. Santos, J. Croes, W. Desmet, and M. M. da Silva, "On redundancy resolution and energy consumption of kinematically redundant planar parallel manipulators," Robotica, vol. 36, no. 6, pp. 809-821, 2018.

[42] S. J. Yan, S. K. Ong, and A. Y. C. Nee, "Stiffness analysis of parallelogram-type parallel manipulators using a strain energy method," Robotics and Computer-Integrated Manufacturing, vol. 37, pp. 13-22, 2016.

[43] E. Cavallo, R. C. Michelini, and V. F. Filaretov, "Conceptual design of an AUV equipped with a three degrees of freedom vectored thruster," Journal of Intelligent and Robotic Systems, vol. 39, no. 4, pp. 365-391, 2004.

[44] L. Nurahmi, J. Schadlbauer, S. Caro, M. Husty, and P. Wenger, "Kinematic analysis of the 3-RPS cube parallel manipulator," Journal of Mechanisms and Robotics, vol. 7, no. 1, Article ID 011008, 2015.

[45] A. Nayak, T. Stigger, M. L. Husty, P. Wenger, and S. Caro, "Operation mode analysis of 3-RPS parallel manipulators based on their design parameters," Computer Aided Geometric Design, vol. 63, pp. 122-134, 2018.

[46] J. Gallardo, H. Orozco, and J. M. Rico, "Kinematics of 3-RPS parallel manipulators by means of screw theory," The International Journal of Advanced Manufacturing Technology, vol. 36, no. 5-6, pp. 598-605, 2008.

[47] N. M. Rao and K. M. Rao, "Multi-position dimensional synthesis of a spatial 3-RPS parallel manipulator," Journal of Mechanical Design, vol. 128, no. 4, pp. 815-819, 2006.

[48] H. S. Kim and L.-W. Tsai, "Kinematic synthesis of spatial 3RPS parallel manipulators," in Proceedings of the ASME 2002 International Design Engineering Technical Conferences and Computers and Information in Engineering Conference, pp. 873-880, 2002.

[49] A. Nayak, L. Nurahmi, P. Wenger, and S. Caro, "Comparison of 3-RPS and 3-SPR parallel manipulators based on their 
maximum inscribed singularity-free circle," in New Trends in Mechanism and Machine Science, pp. 121-130, Springer, Berlin, Germany, 2017.

[50] Q. Li, J. n. Xiang, X. Chai, and C. Wu, "Singularity analysis of a 3-RPS parallel manipulator using geometric algebra," Chinese Journal of Mechanical Engineering, vol. 28, no. 6, pp. 1204$1212,2015$.

[51] D. Thompson, D. Caress, H. Thomas, and D. Conlin, "MBARI mapping AUV operations in the gulf of California 2015," in Proceedings of the OCEANS 2015-MTS/IEEE Washington, Washington, DC, USA, October 2015.

[52] A. Bhattacharyya and S. Steen, "Influence of ducted propeller on seakeeping in waves," Ocean Engineering, vol. 91, pp. 243-251, 2014.

[53] M. Díaz-Rodríguez, J. A. Carretero, and R. Bautista-Quintero, "Solving the dynamic equations of a 3-PRS parallel manipulator for efficient model-based designs," Mechanical Sciences, vol. 7, no. 1, p. 9, 2016.

[54] J. A. Carretero, R. P. Podhorodeski, M. A. Nahon, and C. M. Gosselin, "Kinematic analysis and optimization of a new three degree-of-freedom spatial parallel manipulator," Journal of Mechanical Design, vol. 122, no. 1, pp. 17-24, 2000.

[55] J. Carretero, M. Nahon, and R. Podhorodeski, "Workspace analysis and optimization of a novel 3-DOF parallel manipulator," International Journal of Robotics and Automation, vol. 15 , no. 4, pp. 178-188, 2000.

[56] R. McEwen and K. Streitlien, "Modeling and control of a variable-length AUV," in Proceedings of the 12th International Symposium on Unmanned Untethered Submersible Technology, Durham, NJ, USA, 2001. 